\title{
A Multi-factor Uzawa Growth Theorem and Endogenous Capital-Augmenting Technological Change*
}

\author{
Gregory Casey and and Ryo Horii ${ }^{\mathrm{b}}$ \\ ${ }^{a}$ Economics Department, Williams College, Williamstown, MA, USA \\ ${ }^{\mathrm{b}}$ Institute for Social and Economic Research, Osaka University, Osaka, Japan
}

Version February 16, 2019; Work in progress...

\begin{abstract}
We prove a generalized, multi-factor version of the Uzawa steady state growth theorem. In the two-factor case, the theorem implies that a neoclassical growth model cannot be simultaneously consistent with empirical evidence on both capital-augmenting technical change and the elasticity of substitution between labor and reproducible capital. In the multi-factor case, balanced growth with capital-augmenting technical change is possible as long as capital has a unitary elasticity of substitution with any single non-reproducible factor, significantly increasing the likelihood that neoclassical models can be consistent with empirical findings. To illustrate the importance of this result, we also build a three-factor growth model with endogenous and directed technical change and show that is has a locally stable balanced growth path with a strictly positive rate of capital-augmenting technical change.
\end{abstract}

\section{Introduction}

The neoclassical growth model (NGM) was developed to explain a set of stylized macroeconomic facts that can be classified under the umbrella of balanced growth (Solow, 1956, 1994). As conventionally understood, the Uzawa (1961) steady state growth theorem says that on the balanced growth path (BGP) of a neoclassical growth model, all technological change must be laboraugmenting, unless the production function is Cobb-Douglas (Jones, 2005; Acemoglu, 2008; Jones and Scrimgeour, 2008). This creates a significant problem for the NGM, because data from the

\footnotetext{
${ }^{*}$ The authors are grateful to Been-Lon Chen, Oded Galor, Andreas Irmen, Ping Wang, David Weil, and seminar participants at Brown University, Chulalongkorn University, GRIPS, Kobe University, Shiga University, Osaka University, Tongji University, for their helpful comments and suggestions. This study was financially supported by the JSPS Grant-in-Aid for Scientific Research (15H03329, 15H05729, 16K13353, 17K03788). Any remaining errors are our own.
} 
United States strongly suggest that (i) there is capital-augmenting technical change on the BGP and (ii) the aggregate production function in not Cobb-Douglas (see, e.g., Klump et al., 2007; Oberfield and Raval, 2014). The evidence for capital-augmenting technical change comes from the falling relative price of capital goods (Greenwood et al., 1997; Grossman et al., 2017). ${ }^{1}$

The standard NGM assumes that there are only two factors of production, labor and reproducible capital. In reality, there are many other factors of production, including various types of land, energy, and materials. These factors do not fit well the notion of capital in the NGM in that they cannot be readily accumulated (or reproduced) through savings. In this paper, we examine whether incorporating more factors of production makes it possible for neoclassical models to be consistent with the empirical regularities mentioned above. We prove a generalized version of the Uzawa (1961) steady state growth theorem that incorporates an arbitrary number of factors. We also prove several related propositions and discuss the implications of the theorem in detail, highlighting the difference between the true underlying production function and alternate representations of that function which are the focus of the theorem. We also formulate and analyze an endogenous growth model with three factors of production and directed technical change, highlighting how the theoretical results can be implemented in a tractable framework.

Our results are fairly optimistic for the ability of the neoclassical growth model to incorporate K-augmenting technical change on the BGP. In somewhat colloquial terms, the theorem says that it is possible to have capital-augmenting (K-augmenting) technical change on the BGP as long as there is one non-reproducible factor that has a Cobb-Douglas relationship with reproducible capital. Given the vast number of additional factors that exist in reality, it seems fairly likely that at least one has this desirable property of unit-elastic substitution with capital. To have K-augmenting technology on the BGP, the generalized Uzawa theorem implies that there must be a specific loglinear relationship between the growth rates of the factors of production and the factor-augmenting technologies. We show that this is the endogenous outcome in a model of directed technical change calibrated to U.S. data. Thus, this log-linear relationship does not impose any extra restrictions on the underlying parameter space that is consistent with the data. We discuss our results with greater precision below.

Consider a standard neoclassical environment with an arbitrary number of factors of production and capital that is accumulated linearly from saved output. Suppose that there is a time-varying function that maps aggregate inputs to aggregate output (i.e., an aggregate production function exists). Then, on any BGP, it is possible to represent the relationship between aggregate inputs and aggregate output using a time-invariant function that captures all productivity improvements through factor-augmenting terms on factors other than reproducible capital (Proposition 1). We call this the Uzawa Representation of the BGP. In addition, we demonstrate that it has the same derivatives as the true production function on the BGP as long as factor shares are constant (Propo-

\footnotetext{
${ }^{1}$ See Section 2 for more detail.
} 
sition 2), which guarantees that the Uzawa representation can be used as a local approximation of the original production function. ${ }^{2}$ In the two-factor case, this result has given rise to the conventional wisdom that the Uzawa theorem rules out capital-augmenting technical change on the BGP. The Uzawa theorem, however, is not informative about the actual underlying production function, and it does not establish the uniqueness of the Uzawa Representation. Thus, it does not actually preclude the existence of capital-augmenting technical change on the BGP.

After proving this generalized version of the Uzawa steady state growth theorem, we examine conditions under which it is possible to represent the relationship between inputs and output via a function that has capital-augmenting technical change on the BGP. As explained above, this is necessary if we want to find a function that is able to match data on the relative price of capital goods. We confine our attention to mappings that are time-invariant but for factor-augmenting terms. We call such mappings Factor-Augmenting representations. We find that if there is any single factor of production that can be combined with reproducible capital with unit elasticity, then there are a continuum of different Factor-Augmenting Representations of the mapping from inputs to outputs that have positive growth rates of capital-augmenting technical change (Proposition 3). Moreover, all of these representations have the same derivatives as the true production function as long as factor shares are constant (Proposition 4). In the two factor case, this result simply says that capital-augmenting technical change is possible with Cobb-Douglas production, because capitaland labor-augmenting technical change are essentially equivalent. This is not very helpful when it comes to matching data, however, because empirical studies reject the existence of two-factor Cobb-Douglas production functions. The multi-factor case, however, is much more optimistic. In reality, there are many factors of production besides labor and reproducible capital. For example, there are many types of land, energy, and materials used in production. If any of these has a Cobb-Douglas relationship with reproducible capital, then it is possible to represent output via a function that is consistent with data on both (i) the non-unitary elasticity of substitution between capital and labor and (ii) the falling relative price of capital goods.

In all of the Factor-Augmenting Representations discussed above, there is a specific log-linear relationship between different types of technology that must hold on the BGP. In a world with exogenous technical change, this log-linear relationship between technologies would pose an extra restriction on the nature of production. Our generalized Uzawa theorem, however, is broad enough to incorporate endogenous technical change. To show that the log-linear technology relationship is not actually a restrictive condition for growth models to incorporate capital-augmenting technical change on the BGP, we build a three-factor endogenous growth model with directed technical change and demonstrate that the log-linear technology relationship is the endogenous long-run outcome. We accomplish this goal in two steps. First, we present and analyze an analytic model

\footnotetext{
${ }^{2}$ We are the first to prove these results when there are more than two factors of production. See, Acemoglu (2008) for a similar result in the two-factor case.
} 
where the BGP has a positive rate of capital-augmenting technical change. The dynamical system has three state variables. To examine the stability of the system, we calibrate the model to longterm macroeconomic data from the U.S. We then demonstrate that the calibrated model is both globally and locally stable for a wide range of parameter values. This result guarantees that the log-linear technological relationship is satisfied in the long run regardless of the initial state.

Our results have broad implications for the study of economic growth. It is obviously beneficial in many settings to have a growth model that is consistent with the data on technological progress and the elasticity of substitution between capital and labor. For example, incorporating two (or more) types of technology will allow growth-, development-, and business-cycle accounting exercises to provide a richer description of economy, while still being consistent with balanced growth. Moreover, models with both capital- and labor-augmenting technical change can provide a more nuanced look into how technological progress affects the labor share of income and labor market outcomes.

Contribution and Related Literature. - This paper is related to a long literature on balanced growth and the Uzawa steady state growth theorem. Although the theorem is well known, Uzawa (1961) does not provide a clear statement of proof of the theorem. A simple and intuitive proof was proposed by Schlicht (2006) and updated by Jones and Scrimgeour (2008), Acemoglu (2008), and Irmen (2016). We contribute to this literature by extending the theorem to multiple factors of production and a wider class of neoclassical models. We also provide a more detailed discussion of the theorem and its implication.

The growth literature has long considered the Uzawa theorem to be overly restrictive (Jones and Scrimgeour, 2008). As a result, a second strain of the literature has examined how endogenous technical change might lead an economy to conform to the conditions of the theorem. For example, Acemoglu (2003) and Irmen and Tabaković (2017) present models of directed technical change where the economy endogenously converges to a steady state with only labor-augmenting technical change. Similarly, Jones (2005) and Leon-Ledesma and Satchi (2016) demonstrate conditions under which Cobb-Douglas production is the endogenous outcome of firms choosing between technologies. We build on this work and construct a directed technical change model with where technological progress endogenously conforms to the restrictions generated by the multi-factor Uzawa theorem. Unlike existing work, our model is consistent with data in that it has capital-augmenting technical change on the BGP and an elasticity of substitution between capital and labor that is different than one.

To the best of our knowledge, Grossman et al. (2017) provide the only other attempt to square the Uzawa steady state growth theorem with data on the elasticity of substitution between capital and labor and the capital-augmenting technical change. They consider a neoclassical model with a specific form of complementarity between physical and human capital. We will explain that their 
results can be understood as a particular case of the 2 -factor Uzawa theorem. ${ }^{3}$ Our results indicate that there is a much wider scope for ways in which the NGM can be made to be consistent with the data on elasticities of substitution and capital-augmenting technical change.

Road map - The remainder of the paper proceeds as follows. Section 2 presents the data motivating this study. Broadly speaking, they suggest that a two factor neoclassical growth model cannot be consistent with aggregate data from the U.S. Moreover, factors other than land and reproducible capital are important in production. Motivated by the evidence, Section 3 proves a generalized, multi-factor version of the Uzawa steady state growth theorem. As in the two factor case, the proposition suggests that it is always possible to represent the aggregate production using a function that has no capital-augmenting technical change. We also show that, when factor shares are constant, this function has the correct derivatives, implying that it is useful for economic analysis. The theorem says nothing about the uniqueness of the Uzawa Representation. So, in Section 4 we prove that it is possible to have a Factor-Augmenting Representation with a positive rate of capital-augmenting on the BGP, as long as there is a single non-reproducible factor that has a unitary elasticity of substitution with reproducible capital. Section 5 summarizes and explain these results, focusing on simple cases and existing literature. Sections 6 and 7 present the endogenous growth model, where firms endogenously choose the speed of three types of factor-augmenting technological change, and show that the economy endogenously converges to a BGP with positive K-augmenting technical change. Section 8 discusses the broader implications of our findings, and Section 9 concludes.

\section{Empirical Motivation}

In this section, we discuss the empirical facts that motivate this study. In particular, we quickly review facts on balanced growth and evidence for the existence of capital-augmenting technical change. The Uzawa (1961) steady state growth theorem suggests that these two sets of facts cannot be reconciled in a standard two-factor growth model. So, we conclude by presenting evidence that other factors, such as land and energy are important in the production process.

The neoclassical growth model, first developed by Solow (1956) and Swan (1956), is the central building block of much contemporary research in economic growth. Such models are designed to explain a set of stylized facts, known as 'balanced' or 'steady' growth (Jones, 2016). The main stylized is that income per capita has grown at a constant rate over long periods of time. Panel (a) in figure 1 presents U.S. data from 1950-2012, which clearly demonstrates this pattern. ${ }^{4}$ It also demonstrates that other macroeconomics aggregates have grown at similar rates to GDP, capturing

\footnotetext{
${ }^{3}$ See subsection 5.2 .

${ }^{4}$ See Papell and Prodan (2014), Jones (2016), and others for longer time series and data on other countries.
} 


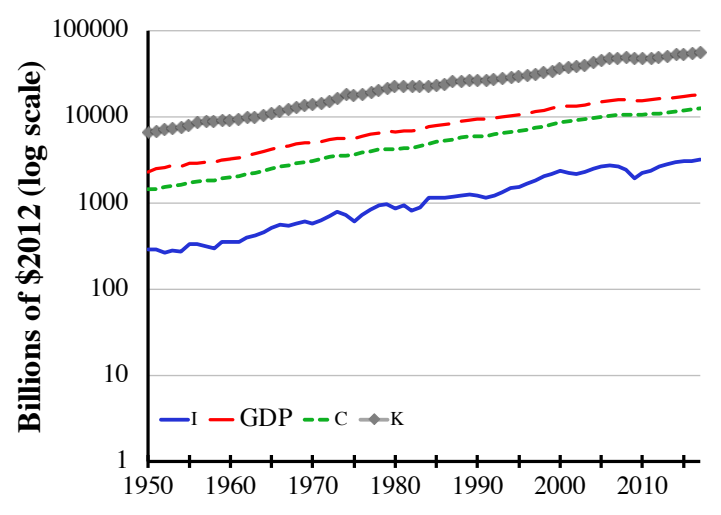

(a) BGP

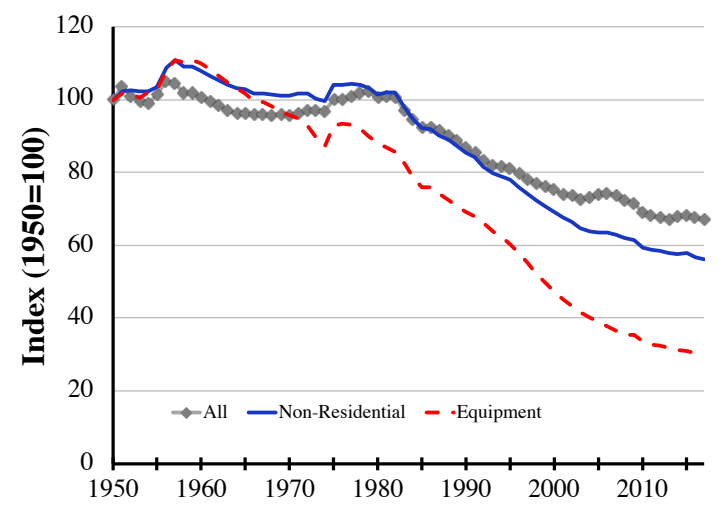

(b) K-Augmenting Technology

Figure 1: Balanced Growth with capital-augmenting technical change. This figures presents some of the main features of balanced growth in the United States. Panel (a) demonstrates the real output, investment, consumption and the capital stock have grown at roughly constant rates over long periods of time. These empirical patterns summarize the notion of balanced growth. Panel (b) demonstrates that the relative price of investment goods, and equipment in particular, having been falling in the United States, even as the economy exhibited the usual signs of balanced growth (see Figure 1.) Falling investment prices is a type of capital-augmenting technical change (Grossman et al., 2017). See appendix section A.1 for details on data sources.

the notion of balance. ${ }^{5}$

To explain these facts, the neoclassical growth model focuses on an aggregate production function that has constant returns to scale in two factors, capital and labor. Intuitively, the key to explaining balanced growth is that capital is reproducible (i.e., it is accumulated from saved output). Thus, capital 'inherits' the constant growth rate of output, implying that the capital-output ratio will be constant in the long-run (Jones and Scrimgeour, 2008). Their joint growth rate is then determined by population growth and technological progress.

The ability of the neoclassical growth model to provide a simple explanation for these facts has led to its widespread adoption (Jones and Romer, 2010). The model, however, relies on some strong assumptions, including those described by the Uzawa (1961) steady state theorem. As conventionally stated, the Uzawa steady state growth theorem is as follows: on a balanced growth path, all technological progress must be labor-augmenting, unless the aggregate production function is Cobb-Douglas. As discussed above, on a balanced growth path with constant factor shares, effective capital and effective labor must grow at the same rate, which must also be the growth rate of output. This is a result of constant returns to scale. Yet, reproducible capital 'inherits' the growth rate of output, which implies that there is no 'space' left for capital-augmenting technical change.

\footnotetext{
${ }^{5}$ As shown in Section 3, the formal definition of balanced growth in the steady state growth theorem only relies on the notion that various macroeconomic aggregates grow at (possibly different) constant rates. In this sense, the constant labor share and capital-output ratio need not be part of our formal definition of balanced growth. Being able to recreate these facts, however, was an important part of the motivation for the original neoclassical model. As we will discuss, they are also important for understanding the implications of theorem.
} 
Cobb-Douglas production is as exception, because capital- and labor-augmenting technological change are essentially equivalent in this special case.

Given the restrictive nature of these conditions for balanced growth, it is natural to ask whether they are consistent with data. A long literature has estimated the elasticity between capital and labor in a two-factor production function and rejected the Cobb-Douglas specification. Most of papers in the literature argue that the elasticity is less than one (Chirinko, 2008). For example, Oberfield and Raval (2014) estimate the macro elasticity of around 0.7 using firm-level micro data, and Antras et al. (2004) estimates an elasticity of 0.6 directly from macro time-series data. ${ }^{6}$

Panel (b) of Figure 1 demonstrates that the relative price of investment goods has been falling in the United States. As discussed in Grossman et al. (2017), this is a type of capital-augmenting technical change. In a setting with perfect competition, decreases in the relative price of capital goods reflect improvement in the efficiency of the investment goods sector. In other words, each unit of forgone consumption is better able to produce output in the future. When we measure capital goods in terms of their value relative to total output, this technological improvement is reflected in the increasing productivity of a measured unit of capital. Section 3 formalizes this intuition, which is also discussed in Grossman et al. (2017). A long literature demonstrates that declining investment prices are a quantitatively important source of growth in the United States (e.g., Greenwood et al., 1997; Krusell, 1998; Krusell et al., 2000). As a result, there is broad consensus that capital-augmenting technical change has been pervasive in the United States over at least the last half a century, even as the economy exhibited signs of balanced growth.

These findings create a puzzle. Given that the elasticity of substitution between capital and labor is not equal to one, the Uzawa theorem implies that any two-factor neoclassical growth model that is consistent with balanced growth is necessarily at odds with evidence on capitalaugmenting technical change. Put differently, the standard neoclassical growth model cannot explain the broader set of stylized growth facts that we observe in the United States.

In this paper, we argue that the inconsistency created by the Uzawa theorem can be rectified by considering additional factors of production, beyond reproducible capital and labor. It obvious that other factors, such as land, energy, and materials, exist in the production process. To highlight their importance, table 1 collects some evidence on the importance of some of these factors in the United States. ${ }^{7}$ Broadly speaking, estimates suggest that non-reproducible factor other than labor account for about $10 \%$ of total factor payments. The rest of this paper examines how our understanding of balanced growth and the Uzawa theorem can be improved by explicitly considering these other non-trivial factors in production.

\footnotetext{
${ }^{6}$ See also, Klump et al. (2007) and Chirinko et al. (2011). Using cross-country data, Karabarbounis and Neiman (2014) find an elasticity greater than one.

${ }^{7}$ Estimating factor shares for inputs other than labor is notoriously difficult and often requires structural assumptions. Our intention is not to endorse any particular estimate. Instead, we simply note that there is ample evidence that factors other than labor and reproducible capital play a non-negligible role in production.
} 


\begin{tabular}{lcc}
\hline \hline Factor & Share & Source \\
\hline Natural Resources & $8 \%$ & Caselli and Feyrer (2007) \\
Land & $5 \%$ & Valentinyi and Herrendorf (2008) \\
Energy & $8.5 \%$ & Energy Information Administration \\
\hline \hline
\end{tabular}

Table 1: This table presents some estimates of U.S. factor shares for inputs other than reproducible capital and labor. Definitions and methodologies vary. See sources for details.

\section{A Multi-factor Uzawa Theorem}

The aim of this section is to show that the steady-state growth theorem by (Uzawa, 1961, hereafter the Uzawa theorem) extends to multi-factor environments that explicitly consider inputs beyond labor and capital. We will then discuss the implications of the theorem and its limitations. The section concludes with a new proposition that clarifies the condition under which the functional form implied by the Uzawa theorem can be used in economic analysis as a first-order approximation of the original production function.

We start with a description of a neoclassical model, which is defined as broadly as possible in order to incorporate a majority of dynamic macroeconomic models. For readability and consistency with the following sections, we consider a discrete time settings, where $t=0,1,2 \ldots$, but it is straightforward to consider the continuous-time equivalents of the following results.

Definition 1. A multi-factor neoclassical growth model is an economic environment that satisfies:

1. Output, $Y_{t}$, is produced from capital, $K_{t}$, and $J \geq 1$ kinds of other inputs, $\left\{X_{j, t}\right\}_{j=1}^{J}$ :

$$
Y_{t}=F\left(K_{t}, X_{1, t}, \ldots, X_{J, t} ; t\right)
$$

The shape of production function $F(\cdot ; t)$ can change with time $t$. In any $t \geq 0$, it has constant returns to scale $(C R S)$ in all inputs, $K_{t}, X_{1, t}, \ldots, X_{J, t}$, and each input has positive and diminishing marginal products. ${ }^{9}$

2. The amount of capital, $K_{t}$, evolves according to

$$
K_{t+1}=Y_{t}-C_{t}-R_{t}+(1-\delta) K_{t}, K_{0}>0,
$$

where $C_{t}>0$ is consumption, $R_{t} \geq 0$ is expenditure other than capital investment or consumption (e.g., R\&D inputs), and $\delta \in[0,1]$ is the depreciation rate.

\footnotetext{
${ }^{9}$ If we allow $J=0$, the only constant-returns-to-scale production function is in the form of $Y_{t}=A_{K, t} K_{t}$. Although it cannot satisfy decreasing marginal products of its input $\left(K_{t}\right)$, this $A K$ functional form obviously satisfies the Uzawa theorem.
} 
Here, $Y_{t}-C_{t}-R_{t}$ in (2) represents physical capital investment. There are a number of points to note regarding Definition 1 . First, if $J$ equals 1 and $X_{1, t}$ is interpreted as labor, $L_{t}$, equation (1) reduces to a familiar two-factor neoclassical production function, $Y_{t}=F\left(K_{t}, L_{t} ; t\right)$. In addition, if we assume $L_{t}$ grows exogenously, Definition 1 essentially coincides with the definition of a neoclassical growth model in Schlicht (2006) and Jones and Scrimgeour (2008), who provide a simple statement and proof of the two-factor Uzawa theorem. ${ }^{10}$

Second, the only reason why capital, $K_{t}$, is distinguished from other production factors $X_{1, t}, \ldots, X_{J, t}$ is that we explicitly specify its accumulation process as in (2), which guarantees that $K_{t}$ can be accumulated linearly with the output. ${ }^{11}$ We will show that the Uzawa theorem holds regardless of the evolution process for other inputs.

Third, we allow for the term $R_{t}$ term in (2). If we set $R_{t}=0$, equation (2) is in line with the previous definitions of the Uzawa theorem. This term is not essential for the proof of the Uzawa Theorem itself, but this generalization accommodates the possibility of endogenous growth, as we examine in later sections. In production function (1), any technological change is captured by the last $t$ term. If we think technology can be affected by the $R \& D$ expenditure, then such expenditure would be included in $R_{t}$ in (2). Similarly, the evolution of other factors $X_{j, t}$ (including population $L_{t}$ ) can be either exogenous or dependent on particular types of expenditure, such as child-raising costs. Such costs are also be included in $R_{t}$.

Fourth, we measure the amount of capital by its value in terms of final output, not by its productivity. Specifically, equation (2) implicitly normalizes the unit of period $t+1$ capital so that period $t$ final output to can be converted to the same units of period $t+1$ capital. However, there are models where the amount of capital is measured by productivity. In particular, Greenwood et al. (1997) and Grossman et al. (2017) assumed that $q_{t+1}>0$ units of period $t+1$ capital can be produced from period $t$ output, where $q_{t}$ increases over time by investment-specific technological change. Definition 1 can accommodate such an extension by a change of variables. Suppose that, instead of (2), physical capital accumulates according to

$$
\breve{K}_{t+1}=\left(Y_{t}-C_{t}-R_{t}\right) q_{t+1}+(1-\breve{\delta}) \breve{K}_{t}
$$

and the production function is given by $Y_{t}=\breve{F}\left(\breve{K}_{t}, \cdot ; t\right)$. Suppose that the growth factor of

\footnotetext{
${ }^{10}$ Grossman et al. (2017) considered a production function with three inputs: capital, labor, and schooling. However, as we discuss in 5.2, it is a particular case of two-factor neoclassical model $(J=1)$ because the production function has constant returns to scale only in capital and labor.

${ }^{11}$ Referring to this property, Jones and Scrimgeour (2008) noted "capital inherits the trend of output." Grossman et al. (2017) make the more precise statement "the value of physical capital that is produced from final goods inherits the trend in output" which holds even when the accumulation technology changes through time (although it should be linear at each point in time). From the theoretical viewpoint, $K_{t}$ needs not to be limited to physical capital. it can be any combination of factors that can be accumulated linearly with the output. For example, in the pre-industrial Malthusian economy where population was proportional to output (e.g., Galor and Weil, 2000; Galor, 2011; Ashraf and Galor, 2011), labor should be included in $K_{t}$, not in $X_{j, t}$. See also Li et al. (2016).
} 
investment-specific technology $q_{t+1} / q_{t}$, is constant at $g_{q}>0 .{ }^{12}$ Then, by defining $K_{t} \equiv \breve{K}_{t} / q_{t}$ and $\delta=\left(\breve{\delta}+g_{q}-1\right) / g_{q}$, equation (3) turns out to be identical with equation (2). ${ }^{13}$ Also, the production technology can be interpreted in the form of (1) by defining $F\left(K_{t}, \cdot ; t\right) \equiv \breve{F}\left(q_{t-1} K_{t}, \cdot ; t\right)=\breve{F}\left(\breve{K}_{t}, \cdot ; t\right)$. In this definition of $F\left(K_{t}, \cdot ; t\right)$, the effect of investment-specific technological change $q_{t-1}$ is captured by the time dependence of $F\left(K_{t}, \cdot ; t\right)$ (i.e., as a part of technological change).

We now define the balanced growth path.

Definition 2. A balanced growth path (BGP) in a multi-factor neoclassical growth model is a path along which all quantities, $\left\{Y_{t}, K_{t}, X_{1, t}, \ldots, X_{J, t}, C_{t}, R_{t}\right\}$, grow at constant exponential rates for all $t \geq 0$. On the BGP, we denote the growth factor of output by $g \equiv Y_{t} / Y_{t-1}$, and the growth factors of any variable $Z_{t} \in\left\{K_{t}, X_{1, t}, \ldots, X_{J, t}, C_{t}, R_{t}\right\}$ by $g_{Z} \equiv Z_{t} / Z_{t-1}{ }^{14}$

Definition 3. A non-degenerate balanced growth path is a $B G P$ with $g_{K}>1-\delta$.

From (2), condition $g_{K}>1-\delta$ means that physical capital investment $Y_{t}-C_{t}-R_{t}$ is strictly positive along the balanced growth path. The rest of the paper focuses on this non-trivial case. We call it a non-degenerate BGP and simply mention it as a BGP when there is no risk of confusion. Note that, while a BGP requires variables to grow at constant rates, it does not require them to grow at the same rate. Still, the following lemma confirms that capital and consumption need to grow at the same speed as output to maintain a BGP.

Lemma 1. On any non-degenerate BGP in a multi-factor neoclassical growth model, capital-output ratio $K_{t} / Y_{t}$ and consumption-output ratio $C_{t} / Y_{t}$ are constant and strictly positive.

Proof. Using the notation in Definition 2, (2) can be written as $K_{0} g_{K}^{t+1}=Y_{0} g^{t}-C_{0} g_{C}^{t}-R_{0} g_{R}^{t}+$ $(1-\delta) K_{0} g_{K}^{t}$. Dividing all terms by $g^{t}$ and rearranging them gives

$$
Y_{0}=C_{0}\left(g_{C} / g\right)^{t}+R_{0}\left(g_{R} / g\right)^{t}+K_{0}\left(g_{K}+\delta-1\right)\left(g_{K} / g\right)^{t} .
$$

Because all three terms on the right hand side (RHS) of (4) are non-negative exponential functions of $t$, every one of them needs to be constant for the sum of all the terms to become constant $\left(Y_{0}\right)$. For the first term $C_{0}\left(g_{C} / g\right)^{t}$ to be constant, $g_{C}=g$ must hold since $C_{0}>0$ from Definition 1 . This means $C_{t} / Y_{t}=C_{0} / Y_{0}>0$. For the third term $\left(g_{K}+\delta-1\right)\left(g_{K} / g\right)^{t}$ to be constant, $g_{K}=g$ must hold since $K_{0}>0$ and $g_{K}>1-\delta$. This implies $K_{t} / Y_{t}=K_{0} / Y_{0}>0 .{ }^{15}$

\footnotetext{
${ }^{12}$ While, for simplicity, we assume $q_{t+1} / q_{t}$ to be constant, it only needs to be constant on the BGP for the purpose of proving the Uzawa theorem.

${ }^{13}$ Note that $\delta=\left(\breve{\delta}+g_{q}-1\right) / g_{q}$ means $1-\breve{\delta}=(1-\delta) q_{t+1} / q_{t}$. Using this, $K_{t+1}=\breve{K}_{t+1} / q_{t+1}=\left(Y_{t}-C_{t}-R_{t}\right)+$ $(1-\breve{\delta}) \breve{K}_{t} / q_{t+1}=\left(Y_{t}-C_{t}-R_{t}\right)+(1-\delta) K_{t}$, which coincides with $(2)$. In this representation, $K_{t} \equiv \breve{K}_{t} / q_{t}$ represents the total value of period $t$ capital in terms of the previous period's final goods. If $g_{q}>1$ (positive investment-specific technological change), the depreciation rate $\delta$ should be higher than $\breve{\delta}$ because older capital experiences obsolescence in addition to physical depreciation.

${ }^{14}$ For simplicity of exposition, we use the growth factor, rather than the growth rate. The conventional period growth rate is obtained by subtracting 1 from the growth factor.

${ }^{15}$ Similarly, we can show $g_{R}=g$ if $R_{0}>0$.
} 
Based on this lemma, we are ready to present a multi-factor version of the Uzawa Theorem.

Proposition 1. (a Multi-Factor Uzawa Theorem) Consider a non-degenerate BGP in a multifactor neoclassical growth model, and define $\widetilde{A}_{X j, t} \equiv\left(g / g_{X j}\right)^{t}$ where $j=1, \ldots, J$. Then, on the BGP,

$$
Y_{t}=\widetilde{F}\left(K_{t}, \widetilde{A}_{X 1, t} X_{1, t}, \ldots, \widetilde{A}_{X J, t} X_{J, t}\right) \text { holds for all } t \geq 0
$$

where $\widetilde{F}(\cdot) \equiv F(\cdot ; 0)$.

Proof. From the definition of $\widetilde{A}_{X j, t} \equiv\left(g / g_{X j}\right)^{t}$, the growth factor of $\widetilde{A}_{X j, t} X_{j, t}$ is $g$ for all $j$. The growth factor of $K_{t}$ is also $g$ from Lemma 1 . Therefore, all the arguments in function $\widetilde{F}(\cdot)$ are multiplied by $g$ each period. This means that the RHS of (5) is multiplied by $g$ each period since $\widetilde{F}(\cdot) \equiv F(\cdot ; 0)$ has constant returns to scale. Note that in period 0 , equation (5) holds true because it is identical with (1). Therefore, (5) holds for all $t \geq 0$, where the both sides are multiplied by $g$ in every period.

This proposition states that the conventionally-known Uzawa theorem applies to multi-factor environments. While the Uzawa theorem applies to quite a wide array of macroeconomic models, it is important to understand what the theorem does and does not imply. Recall that the neoclassical production function $F(\cdot ; t)$ in $(1)$ is a time-varying function that potentially depends on $t$ in complex ways. However, if a BGP exists, the Uzawa theorem says that there should be a simple representation of this dependence of function $F(\cdot ; t)$ on $t$, which holds at least along this particular BGP. We call this representation, given by (5), the Uzawa representation, which consists of a time-invariant function $\widetilde{F}(\cdot)$ along with exponentially growing $\widetilde{A}_{X j, t}$ terms.

Caution is needed when interpreting $\widetilde{F}(\cdot)$ as a production function, because (5) is not a functional relationship. Proposition 1 only guarantees that the value of $\widetilde{F}(\cdot)$ coincides with that of the original production function $F(\cdot ; t)$ on a particular BGP. As is clear from the proof of the proposition, function $\widetilde{F}(\cdot)$ contains no information about what will happen when inputs deviate even slightly from the BGP. When the amount of one of the factors is changed from the BGP value, equation (5) does not hold in general. In this sense, the Uzawa Theorem does NOT say that $\widetilde{F}(\cdot)$ in (5) is a production function.

Moreover, there is no guarantee that the derivatives of function $\widetilde{F}(\cdot)$, even on the BGP, are equal to the derivatives of the production function $F(\cdot ; t)$, apart from time $t=0$. This is inconvenient because it implies that the Uzawa representation is unusable for any marginal analysis. Thus, we now provide a condition under which the Uzawa representation has the "right" first-order derivatives.

Proposition 2. (Derivatives of the Uzawa representation) Let $F_{Z}(\cdot ; t)$ denote the partial 
derivative of function $F(\cdot ; t)$ with respect to its argument $Z \in\left\{K_{t}, X_{1, t}, \ldots, X_{J, t}\right\} .{ }^{16}$ If the share of factor $Z$, i.e., $s_{Z}=F_{Z}(\cdot ; t) Z_{t}$, is constant on a non-degenerate BGP of a multi-factor neoclassical growth model, then the following holds on the BGP:17

$$
\frac{\partial}{\partial Z_{t}} \widetilde{F}\left(K_{t}, \widetilde{A}_{X 1, t} X_{1, t}, \ldots, \widetilde{A}_{X J, t} X_{J, t}\right)=F_{Z}\left(K_{t}, X_{1, t}, \ldots, X_{J, t} ; t\right) \text { for all } t \geq 0 \text {. }
$$

Proof. See appendix section A.2.

If the factor shares are constant on the BGP, as implied by Kaldor (1961) Facts ${ }^{18}$ in the case of two factors, (6) says that $\widetilde{F}(\cdot)$ has the same derivatives as the original production function $F(\cdot ; t)$ on the BGP, justifying its use in marginal analyses. ${ }^{19}$

Equally importantly, Propositions 1 and 2 jointly imply that the Uzawa representation is a first-order approximation of the original production function around the BGP if factor shares on the BGP are constant. This result legitimizes the use of production functions in the form of the Uzawa representation in many applications, such as the business cycle research, where variables fluctuate along a BGP. ${ }^{20}$

\section{Factor Augmenting Representations}

The particular form of the Uzawa representation invites us to interpret the time dependence of production function in terms of factor-augmenting technological change. By viewing the $\widetilde{A}_{X j, t}$ 's in the Uzawa representation (5) as the factor $X_{j, t}$-augmenting technology terms, Proposition 1 implies that it is always possible to interpret the time variation of the original production function $F(\cdot ; t)$ on the BGP in terms of exponential augmentation of production factors. Then, it is tempting to conclude that there should be no technological change that enhances the productivity of capital on the BGP, because there is apparently no $\widetilde{A}_{K, t}$ term in (5).

However, this reasoning is insufficient, because Proposition 1 does not establish uniqueness. As a result, it does not rule out the existence of factor-augmenting representations of the original

\footnotetext{
${ }^{16}$ More precisely, $F_{K}(\cdot ; t)$ denotes the partial derivative of function $F(\cdot ; t)$ with respect to its first argument, whereas $F_{X j}(\cdot ; t)$ denotes the partial derivative of $F(\cdot ; t)$ with respect to its $1+j$ th argument.

${ }^{17}$ Following the convention in economics, $\frac{\partial}{\partial X_{j, t}} \widetilde{F}(\cdot)$ represents the partial derivative with respect to variable $X_{j, t}$, which is the marginal product of factor $X_{j, t}$ given $\widetilde{F}(\cdot)$ is the production function. This should not be confused with $\widetilde{F}_{X_{j}}(\cdot)$, which represents the partial derivative of function $\widetilde{F}_{X_{j}}(\cdot)$ with respect to its $(1+j)$ th argument. They differ from each other when the argument of the function is not just a variable. For example, using the chain rule, $\frac{\partial}{\partial X_{j, t}} \widetilde{F}\left(K_{t}, \widetilde{A}_{X 1, t} X_{1, t}, \ldots, \widetilde{A}_{X J, t} X_{J, t}\right)=\widetilde{A}_{X j, t} \widetilde{F}_{X_{j}}\left(K_{t}, \widetilde{A}_{X 1, t} X_{1, t}, \ldots, \widetilde{A}_{X J, t} X_{J, t}\right)$

${ }^{18}$ See also the discussion in Jones and Romer (2010).

${ }^{19}$ See Acemoglu (2008) for a related results in the two-factor case.

${ }^{20}$ In the previous studies on the Uzawa theorem, there seems to be no consensus whether the constancy of factor shares was a requirement for the Uzawa theorem or not. The original Uzawa (1961) paper assumed constant factor shares, but others such as Schlicht (2006) did not require them. Proposition 1 and 2 clearly show that constant factor shares are not required for the conventionally-known part of the Uzawa theorem, but they are needed for interpreting the Uzawa presentation as an approximated production function.
} 
production function other than the Uzawa representation. In this section, we explore the possibility that the original production function has multiple factor-augmenting representations, where the Uzawa representation is just one possibility. Let us start by defining what constitutes a factoraugmenting representation.

Definition 4. A Factor-Augmenting Representation of the original production function (1) is a combination of a time-invariant constant-returns-to-scale function $F^{A U G}(\cdot)$ and the growth factors of factor-augmenting technologies $\gamma_{K}>0$ and $\gamma_{X j}>0, j \in\{1, \ldots, J\}$, such that the paths of output and inputs on a BGP satisfy

$$
Y_{t}=F^{A U G}\left(A_{K, t} K_{t}, A_{X 1, t} X_{1, t}, \ldots, A_{X J, t} X_{J, t}\right) \text { holds for all } t \geq 0,
$$

where $A_{K, t}=\left(\gamma_{K}\right)^{t}$ and $A_{X j, t}=\left(\gamma_{X j}\right)^{t}$.

By comparing (5) with (7), it is clear that the Uzawa representation constitutes a factoraugmenting representation. However, (5) focuses only on a certain special case where all effective factors grow at the same rate of $g$, while (7) permits different growth rates among separate effective factors. In other words, the Uzawa representation hypothesizes that there is no factor substitution taking place when the economy grows along the BGP. The homothetic expansion of every effective input is the simplest interpretation of a steadily growing economy, but it does not necessarily constitute the best description of the reality.

To see this, suppose that every effective input, including effective capital, grows at the same speed as the output. Recall that the physical capital is already growing at the same speed as output on the BGP (from Lemma 1). Then, there is no room for additional capital-augmenting technological progress to further augment its effectiveness. (This is a well-known property of the Uzawa Theorem). However, as discussed in Section 2, there is clear evidence that the productivity of capital, measured in terms of output as in our model, has steadily been increasing for a long time. Therefore, the interpretation of the BGP as being a homothetic expansion of every input is at odds with a well-established stylized fact.

This contradiction leads us to consider a broader range possibilities in which effective inputs grow at different constant rates. For such a possibility to constitute a BGP, output must also grow at a constant rate. To illustrate this point, note that the growth rate of output in the factor-augmenting representation (7) can approximately be written as follows: ${ }^{21}$

$$
Y_{t+1} / Y_{t} \approx s_{k, t} \gamma_{K} g_{K}+\sum_{j=1}^{J} s_{X j, t} \gamma_{X j} g_{X j},
$$

\footnotetext{
${ }^{21}$ This decomposition is obtained by Taylor-expanding the RHS of (7) for $t+1$ with respect to every effective factor, around the period $t$ values for the variables, and divide the result by the RHS of (7) for $t$. The Taylor-expansion is exact when the variables in $t$ and $t+1$ are sufficiently close, or equivalently, in continuous time.
} 
where $s_{k, t}$ is the share of capital at time $t$ and similarly for $s_{X j, g}$. In the Uzawa representation, $\gamma_{K} g_{K}=\gamma_{X j} g_{X j}$ holds for all $j$. Therefore, as long as the production function is constant returns to scale (which guarantees $s_{K, t}+\sum_{j=1}^{J} s_{X j, g}=1$ ), the RHS is always constant, even if factor shares are not constant. In contrast, if the effective factors grows at different speed, i.e., when $\gamma_{K} g_{K}$ and $\gamma_{X j} g_{X j}$ 's are different, the RHS of (8) becomes stationary only when the factor shares, $s_{k, t}$ and $s_{X j, g}$ 's, remain constant over time.

This raises an important question: can factor shares be constant when factor substitution is taking place on the balanced growth path? The answer is yes, as long as some of the effective factors are substitutable to each other with the unitary elasticity of substitution. Below, we formally establish this conjecture. We must first define the elasticity of substitution when there are more than two inputs. ${ }^{22}$

Definition 5. The Elasticity of Substitution between capital $K_{t}$ and input $X_{j}$ in multi-factor neoclassical production function $F\left(K, X_{1}, \ldots, X_{J} ; t\right)$ in $(1)$ is defined by

$$
\sigma_{K X j, t}=-\left.\frac{d \ln \left(K / X_{j}\right)}{d \ln \left(F_{K}\left(K, X_{1}, \ldots, X_{J} ; t\right) / F_{X j}\left(K, X_{1}, \ldots, X_{J} ; t\right)\right)}\right|_{Y, \mathbf{X}_{-j}: \text { const }},
$$

where $\mathbf{X}_{-j} \equiv\left\{X_{1}, \ldots, X_{J}\right\} \backslash X_{j}$ represents the inputs other than $K$ and $X_{j}$.

Let us consider explicitly many factors of production other than capital, $\left\{X_{1, t}, \ldots, X_{J, t}\right\}$, and suppose that some of them (e.g., land or energy) are substitutable with capital $K_{t}$ with unitary elasticity in the period 0 production function, $F(\cdot ; 0) .{ }^{23}$ In that case, without a loss of generality, we reorder these factors so that the first $j^{*} \in\{0, \ldots, J\}$ of them can be substituted with capital with the unitary elasticity of substitution. ${ }^{24}$ Then, production in period 0 can be rewritten in the following nested form:

Lemma 2. Suppose that $\sigma_{K X j, 0}=1$ for $j=1, \ldots, j^{*}$. Then, there exist parameters $\alpha>0$ and $\xi_{j}>0, j \in\{1, \ldots, J\}$, such that $\alpha+\sum_{j=1}^{j^{*}} \xi_{j}=1$ and

$$
Y_{0}=\widehat{F}\left(K_{0}^{\alpha} \prod_{j=1}^{j^{*}}\left(X_{j, 0}\right)^{\xi_{j}}, X_{j^{*}+1,0}, \ldots, X_{J, 0}\right),
$$

\footnotetext{
${ }^{22}$ When there are more than two production factors, there are various ways to define the elasticity of technical substitution (See, Stern 2011 Journal of Productivity Analysis for a concise taxonomy). The elasticity in (9) is calculated in one of the most straightforward settings, where the output and other inputs are kept constant when $X_{j}$ and $K$ are simultaneously changed. It is the inverse of the symmetric elasticity of complementarity (SEC), defined in Stern (2010, Economics Letters), which has a desirable property of symmetry between the two variables.

${ }^{23}$ We only need to assume that the production function has this property at some point in time. We just normalize that time as $t=0$.

${ }^{24}$ When there is no such input, with a slight abuse of notation, let us write $j^{*}=0$.
} 
where $\widehat{F}(\cdot)$ is a constant-returns-to-scale function, defined by

$$
\widehat{F}\left(z_{0}, z_{j^{*}+1}, \ldots, z_{J}\right) \equiv F(z_{0}^{1 / \alpha}, \underbrace{1, \ldots, 1}_{j^{*}}, z_{j^{*}+1}, \ldots, z_{J} ; 0) .
$$

Proof. See appendix section A.2.

Intuitively, if $K_{0}$ is substitutable with factors $\left\{X_{1,0}, \ldots, X_{j^{*}, 0}\right\}$ with unit elasticity, then the production function can be expressed as if they are combined together in the Cobb-Douglas fashion to form an intermediate input $M_{t} \equiv\left(A_{K, t} K_{t}\right)^{\alpha} \prod_{j=1}^{j^{*}}\left(A_{X j, t} X_{j, t}\right)^{\xi_{j}}$. This virtual intermediate input, which we call capital composite, will be placed inside a constant-returns-to-scale function $\widehat{F}(\cdot)$, which has $j^{*}$ fewer arguments than $\widetilde{F}(\cdot) \equiv F(\cdot ; 0)$. Using this nested form, the following proposition shows the set of the possible factor-augmenting representations of a given BGP.

Proposition 3. (Factor-Augmenting Representations of a BGP) Suppose that $\sigma_{K X j, 0}=1$ for $j=1, \ldots, j^{*}$. On a non-degenerate BGP, let constants $\gamma_{K}>0$ and $\gamma_{X j}>0, j \in\left\{1, \ldots, j^{*}\right\}$, be any combination that satisfy

$$
\gamma_{K}^{\alpha} \prod_{j=1}^{j^{*}}\left(\gamma_{X j} g_{X j}\right)^{\xi_{j}}=g^{1-\alpha}
$$

Also let $\gamma_{X j}=g / g_{X j}$ for $j=j^{*}+1, \ldots, J$. Then, on the BGP,

$$
Y_{t}=\widehat{F}\left(\left(A_{K, t} K_{t}\right)^{\alpha} \prod_{j=1}^{j^{*}}\left(A_{X j, t} X_{j, t}\right)^{\xi_{j}}, A_{X j^{*}+1, t} X_{j^{*}+1, t}, \ldots, A_{X J, t} X_{J, t}\right) \text { for all } t \geq 0
$$

where $A_{K, t}=\left(\gamma_{K}\right)^{t}$ and $A_{X j, t}=\left(\gamma_{X j}\right)^{t}, j \in\{1, \ldots, J\}$.

Proof. See appendix section A.2.

Note that (13) constitutes a factor augmenting representation because its RHS is a function of effective factors $\left(A_{K, t} K_{t}\right),\left(A_{X 1, t} X_{1, t}\right), \ldots,\left(A_{X J, t} X_{J, t}\right)$ and it has constant returns to scale in all of these $J+1$ factor-augmented variables.

Proposition 3 can be seen as a generalized version of the multi-factor Uzawa theorem (Proposition 1). When there is no factor that is substitutable with capital with unit elasticity at time 0 (i.e., $j^{*}=0$ ), then Proposition 3 becomes identical with Proposition $1 .^{25}$ However, given that there are many factors of production in reality, it seems highly likely that at least one of them is substitutable with capital with unit elasticity $\left(j^{*} \geq 1\right)$. In this case, there is a continuum of combinations $\left\{\gamma_{K}, \gamma_{X j}\right\}$ that satisfy condition (12), and there always exists a continuum of its subset where $\gamma_{K}>1$. In other words, if $j^{*} \geq 1$, there are a continuum of possible factor-augmenting representations of a BGP, and many of them are with strictly positive capital-augmenting technological

\footnotetext{
${ }^{25}$ If $j^{*}=0$, condition $\alpha+\sum_{j=1}^{j^{*}} \xi_{j}=1$ in Lemma 2 implies $\alpha=1$. Then, condition (12) reduces to $\gamma_{K}=1$, which means $A_{K, t}=1$ for all $t$. Then, (13) becomes identical to (5).
} 
change. The Uzawa representation, where $\gamma_{K}=1$ and $\gamma_{X j}=g / g_{X j}$ for all $j$, is also included in the set of possible factor-augmenting representations. However, this is a special case in that it only constitutes a single point in a continuum of possible factor-augmenting representations. In principle, to distinguish between various factor-augmenting representations, we need more information than just the paths of the output and inputs, such as observations on the productivity growth of capital or other inputs from macroeconomic data, as we discuss in the next section.

We finish this section by confirming that any factor-augmenting representation has the same derivative as the original production function under a condition similar to Proposition 2, and thus can be used as a first-order approximation in economic analyses:

Proposition 4. (Derivatives of the Factor-Augmenting Representation) Suppose that $\sigma_{K X j, 0}=1$ for $j=1, \ldots, j^{*}$ and that the production function at time $t=0$ can be represented in the form of (10). If the share of factor $Z \in\left\{K_{t}, X_{1, t}, \ldots, X_{J, t}\right\}$, i.e., $s_{Z}=F_{Z}(\cdot ; t) Z_{t}$, is constant on a non-degenerate BGP of a multi-factor neoclassical growth model, the following holds on the $B G P$ :

$$
\begin{array}{r}
\frac{\partial}{\partial Z_{t}} \widehat{F}\left(\left(A_{K, t} K_{t}\right)^{\alpha} \prod_{j=1}^{j^{*}}\left(A_{X j, t} X_{j, t}\right)^{\xi_{j}}, A_{X j^{*}+1, t} X_{j^{*}+1, t}, \ldots, A_{X J, t} X_{J, t}\right) \\
=F_{Z}\left(K_{t}, X_{1, t}, \ldots, X_{J, t} ; \text { t for all } t \geq 0 .\right.
\end{array}
$$

Proof. See appendix section A.2.

\section{$5 \quad$ Summary and Examples}

So far, we have established both the wide applicability of the Uzawa theorem and also its limitations, focusing on economic environments specified as loosely as possible so that most dynamic macroeconomic models fit into our framework.

As for the wide applicability, we have shown that if the economy exhibits balanced growth, as observed in many countries, there always exist a representation of the aggregate production func-

tionn $Y_{t}=\widetilde{F}\left(K_{t}, \widetilde{A}_{X 1, t} X_{1, t}, \ldots, \widetilde{A}_{X J, t} X_{J, t}\right)$, which explains the growth process by factor-augmenting technological changes on other factors than capital (Proposition 1). This simple representation, called the Uzawa Representation, explains the balanced growth by homothetic expansion of every production factor. If the factor shares are constant, we have shown that the Uzawa Representation matches the behavior of the actual (unknown) production function not only on the BGP, but also around the BGP (Proposition 2). This means that, with the observation that the economy is on average steadily growing and the factor share are stationary, we can always use a production function in the form of the Uzawa Representation to study long-term growth and fluctuations around the BGP, as long as we believe that the economy can be described by a neoclassical environment, potentially with many production factors. 
While Propositions 1 and 2 are quite strong results, the Uzawa representation fails to explain one critical aspect of growth. Because there is no $\widetilde{A}_{K}$ term in the Uzawa representation, it appears to say that there should be no improvement in the productivity of capital. We have clarified that the Uzawa theorem does not say that the Uzawa representation (without any capital-augmenting technological change) is the only representation that explains the observed balanced growth. To the contrary, our generalized theorem (Proposition 3) constructs a continuum of factor-augmenting representations of the production function. Every candidate representation can explain the observed balanced growth, but they differ in the rates of factor-augmenting technological progress among production factors. This means that it is possible to choose from those candidate representations so that the rate of capital-augmenting technological progress in the model matches the rate observed in data. Proposition 4 guarantees that, if factor shares are constant, the chosen representation can be used as a production function not only for analyzing long-term growth but also for studying economic fluctuations, because the representation constitutes a local approximation of the actual production function along the BGP.

So far, we have presented our results in as general a setting as possible. To incorporate these results into neoclassical models suitable for economic analysis, it is necessasry to consider specific functional forms for the aggregate production function. In the remainder of this section, we present three examples that explore the simplest way to make neoclassical models consistent with aggregate data on the relative price of capital and the elasticity of substitution between capital and labor. In subsection 5.1, we explain why a standard neoclassical economy only with two factors cannot simultaneously be consistent with data on (i) the aggregate elasticity of substitution between capital and labor and (ii) the relative price of capital. Then, subsection 5.2 discusses the approach taken by Grossman et al. (2017) as a special case of the 2-factor neoclassical environment. Finally, subsection 5.3 shows that the conflict between data and neoclassicaly models can be resolved when including factors of production beyond labor and reproducible capital.

\subsection{Standard 2-Factor Neoclassical Growth Model}

Suppose that the original production function $Y_{t}=F\left(K_{t}, L_{t} ; t\right)$ uses only two kinds of inputs, capital, $K_{t}$, and labor, $L_{t}$. Then Proposition 1 says that, on any BGP with positive investment, this production function can always be written as: $Y_{t}=\widetilde{F}\left(K_{t}, A_{L, t} L_{t}\right)$. However, if these two factors are substitutable with unit elasticity $\left(\sigma_{K L}=1\right)$, Proposition 3 shows there are other possible factoraugmenting representations of the same production function: ${ }^{26}$

$$
Y_{t}=\bar{A}\left(A_{K, t} K_{t}\right)^{\alpha}\left(A_{L, t} L_{t}\right)^{1-\alpha}, \text { where } \bar{A}>0 \text { is a constant }
$$

\footnotetext{
${ }^{26}$ When there are two factors $(J=1)$ and they are substitutable with unit elasticity $\left(j^{*}=1\right)$, equation $(13)$ in Proposition 3 implies that $Y_{t}=\widehat{F}\left(\left(A_{K, t} K_{t}\right)^{\alpha}\left(A_{L, t} L_{t}\right)^{1-\alpha}\right)$. Because function $\widehat{F}(\cdot)$ has constant returns to scale and has only one argument, we can write $\widehat{F}(x)=\bar{A} x$ for some $\bar{A}>0$, which gives (15).
} 
which includes an Uzawa representation $Y_{t}=\bar{A} K_{t}^{\alpha}\left(\widetilde{A}_{L, t} L_{t}\right)^{1-\alpha}$ as a special case. Condition (12) implies that the growth factor of technologies, $\gamma_{K}>0$ and $\gamma_{L}>0$, can take any values as long as $\gamma_{K}^{\alpha} \gamma_{L}^{1-\alpha}=g^{1-\alpha}$. By rewriting (15) as $Y_{t}=A_{t} K_{t}^{\alpha} L_{t}^{1-\alpha}$, where the TFP $A_{t}$ is given by $A_{t} \equiv$ $\bar{A} A_{K, t}^{\alpha} A_{L, t}^{1-\alpha}$, it is clear that various combinations of capital- and labor-augmenting technological changes give the same rate of growth for the TFP and, therefore, output.

This result confirms the widely understood version of the Uzawa theorem: on a BGP, all technological progress must be labor-augmenting, unless the production function is Cobb-Douglas. As we have seen in Section 2, this theoretical results is in contradiction with the two stylized findings: (i) the productivity of capital has been steadily increasing, and (ii) the elasticity of substitution between capital and labor is less than unity, ruling out the Cobb-Douglas production function. Any standard, two-factor production function cannot reconcile these two stylized facts.

\subsection{Inclusion of Schooling in a Two-Factor model}

Grossman et al. (2017) propose a possible solution to this contradiction by including schooling, $s_{t} \geq 0$, in a standard two-factor production function. Their result can be understood intuitively in terms of our analytical framework. While they directly started their analysis from a factoraugmenting representation, it is worthwhile to consider an underlying time-varying production function in the form of (1):

$$
Y_{t}=F\left(K_{t}, L_{t} ; t\right)=F^{s}\left(D\left(s_{t}\right)^{a} K_{t}, D\left(s_{t}\right)^{-b} L_{t} ; s_{t}, t\right),
$$

where $a>0, b>0, D(\cdot) \in[0,1]$, and $D^{\prime}(\cdot)<0$. The definition of a neoclassical growth model in Definition 1 is general enough to include function $F^{s}\left(\cdot ; s_{t}, t\right)$ in $(16)$ as a particular case of a two-factor neoclassical production function with $J=1 .^{27}$

When schooling,$s_{t}$, increases, the multiplier $D\left(s_{t}\right)^{a}$ on $K_{t}$ shrinks, raisin the marginal product of capital. The opposite holds for labor. In this way, Grossman et al. (2017) specified a certain type of complementarity between schooling and capital. Note that $s_{t}$ is not a production factor in a neoclassical sense, because the production function has constant returns to scale only in capital and labor.

From Proposition 1, this production function has an Uzawa representation $Y_{t}=\widetilde{F}\left(K_{t}, \widetilde{A}_{L, t} L_{t}\right)$ with $\widetilde{A}_{L}=\left(g / g_{L}\right)^{t}$ on a BGP, where both effective factors $K_{t}$ and $\widetilde{A}_{L, t} L_{t}$ grow at the same speed as output. Therefore, if we directly look at the simplest representation of the BGP, then it would imply no capital-augmenting technological progress. Instead, Grossman et al. (2017) interpret the

\footnotetext{
${ }^{27}$ The time-varying effects of $D\left(s_{t}\right)$ and $s_{t}$ itself on output $Y_{t}$ in (16) are captured by the $t$ term in $F\left(K_{t}, L_{t} ; t\right)$. Note also that Grossman et al. (2017) considered investment-specific technological change, which can be rewritten in the form of Definition 1 as we discussed in Section 3.
} 
production function in the following way, keeping the multiplier $D\left(s_{t}\right)$ term in the expression:

$$
Y_{t}=\widetilde{F}\left(K_{t}, \widetilde{A}_{L, t} L_{t}\right)=\widetilde{F}\left(A_{K, t} D\left(s_{t}\right)^{a} K_{t}, A_{L, t} D\left(s_{t}\right)^{-b} L_{t}\right) .
$$

Comparing the arguments in the right hand side and these in the middle, we immediately obtain $A_{K, t}=D\left(s_{t}\right)^{-a}$ and $A_{L, t}=\widetilde{A}_{L, t} D\left(s_{t}\right)^{b}$. Because the multiplier $D\left(s_{t}\right)^{a}$ shrinks as $s_{t}$ increases, the capital-augmenting technology $A_{K, t}$ must grow so as to exactly offset the shrinking $D\left(s_{t}\right)^{a}$ term. Conversely, the labor-augmenting term $A_{L, t}$ should grow slower than that in the Uzawa representation $\widetilde{A}_{L, t}$ because the multiplier $D\left(s_{t}\right)^{-b}$ is also augmenting the labor. ${ }^{28}$

Within the limits of the two-factor Uzawa theorem, Grossman et al. (2017) propose a new interpretation of the production function, which provides the first possible solution to the contradiction raised by the Uzawa theorem. It is important to note, however, that schooling must enter the production function precisely in the form of (17), where exactly the same function $D\left(s_{t}\right)$ must appear both before capital and labor, with the powers of opposite signs. In addition, the functional form of $D\left(s_{t}\right)$ and the dynamic path $s_{t}$ in equilibrium must be in such a way that $D\left(s_{t}\right)$ shrinks exponentially over time. Future empirical work could greatly inform our understanding of long-run economic growth by testing whether the formulation (16) is consistent with data. In this paper, we propose a wider class of functions that are consistent with balanced growth. The next subsection discusses a particularly simple example.

\subsection{A Simple Three-Factor Model with Land}

As we have seen in Section 2, a significant portion of GDP is paid to production factors that do not fit well in either the notion of $K_{t}$ or $L_{t}$. Thus, it is natural to consider production functions that are beyond the limits of the two-factor Uzawa theorem.

Adding additional factors of production makes it possible to reconcile the data with a neoclassical model. While the labor cannot be substituted by capital with unitary elasticity $\left(\sigma_{K L} \neq 1\right)$, Proposition 3 only requires that there is a single production factor that is substitutable with capital with unitary elasticity. In this case, there exist factor-augmenting representations of the production function that have capital-augmenting technological change $\left(\gamma_{K}>1\right)$.

Let us consider the simplest extension of the standard neoclassical production function,

$$
Y_{t}=F_{t}\left(K_{t}, L_{t}, X_{t} ; t\right) \text {, where } X_{t}=X_{0} g_{X}^{t} \text { for all } t, X_{0}>0, g_{X}>0 .
$$

\footnotetext{
${ }^{28}$ From these observations, the main result of (Grossman et al., 2017, proposition 2) can easily be obtained as follows. Taking the growth factor of the both sides of $A_{K}=D\left(s_{t}\right)^{-a}$ gives $\gamma_{K}=g_{D}^{-a}$. From this we obtain a discrete time equivalent of their proposition 2(ii): $g_{D}=\gamma_{K}^{-1 / a}$. Note that Grossman et al. (2017) assumed $L_{t}=D\left(s_{t}\right) N_{t}$, which means $g_{L}=g_{D} g_{N}$. Because effective labor $A_{L, t} D\left(s_{t}\right)^{-b} L_{t}$ in (17) must grow at the same rate as output, $g=\gamma_{L} g_{D}^{-b} g_{L}=\gamma_{L} g_{D}^{1-b} g_{N}=\gamma_{L} \gamma_{K}^{(b-1) / a} g_{N}$, which is a discrete time equivalent of their proposition 2(i).
} 
Here, we have a third production factor $X_{t}$, which is either growing $\left(g_{X}>1\right)$, shrinking $\left(g_{X} \in\right.$ $(0,1))$, or constant $\left(g_{X}=1\right)$. One example of such a factor is land. In that case, $g_{X}$ represents the growth factor of the available land space. If the total area of available land asymptotes to an upper bound in the long run, then $g_{X}$ would be one on the BGP. Another example is natural resources. If it is non-renewable, $g_{X} \in(0,1)$ will likely hold, while a renewable energy source (e.g., sun light) could have $g_{X}=1$.

Among many candidates for the third production factor, we focus on those that have a unitary elasticity of substitution with capital in the initial period: $\sigma_{K X, 0}=1$. For concreteness, let us call this factor land. Then, Proposition 3 implies that, along a non-degenerated BGP, the production function can be represented in a factor-augmenting fashion:

$$
Y_{t}=\widehat{F}\left(\left(A_{K, t} K_{t}\right)^{\alpha}\left(A_{X, t} X_{t}\right)^{1-\alpha}, A_{L, t} L_{t}\right), \alpha \in(0,1)
$$

where the growth factor of technology variables can be any combination of $\gamma_{K}>0, \gamma_{X}>0$, and $\gamma_{L}>0$ such that $\gamma_{K}^{\alpha}\left(\gamma_{X} g_{X}\right)^{1-\alpha}=g^{1-\alpha}=\gamma_{L} g_{L}$. Rearranging, we find that in a BGP, the growth factor of capital-augmenting technology must satisfy,

$$
\gamma_{K}=\left(\frac{\gamma_{L} g_{L}}{\gamma_{X} g_{X}}\right)^{(1-\alpha) / \alpha}
$$

Thus, there is a positive capital-augmenting technological change on a BGP $\left(\gamma_{K}>1\right)$, if the effective input of the third factor $A_{X, t} X_{t}$ grows slower than effective labor $A_{L, t} L_{t}$, which grows proportionally to output $Y_{t}$.

This finding raises an important question: even if there is a factor with $\sigma_{X_{j}, K}=1$, will the rates of technological change $\gamma_{K}, \gamma_{X}$, and $\gamma_{L}$ be determined so as to satisfy the log-linear condition (20)? If their values are exogenously given, then this is a knife-edge case. If growth rates are endogenous, however, this need not pose any additional restrictions on the model. In Section 6, we develop a growth model with endogenous and directed technical change and show that $\gamma_{K}, \gamma_{X}$, and $\gamma_{L}$ are endogenously chosen so that condition (20) is satisfied on the BGP. In Section 7, we calibrate a version of the model to moments from the long-term U.S. data and show that the BGP with positive capital-augmenting technical change is stable for a wide range of parameters.

The next section will develop an endogenous growth model where firms endogenously choose the amount of R\&D investments to enhance the productivity of each production factors. We show that in the BGP equilibrium of that model, $\gamma_{K}, \gamma_{X}$, and $\gamma_{L}$ are indeed endogenously chosen, so that condition (20) is satisfied. 


\section{A Full Model with Endogenous Directed Technological Change}

So far, we discussed the implications of the extended Uzawa theorem only focusing on the production sector, without explicitly considering $R \& D$ activities or the demand side of the economy. Now, we develop a complete endogenous growth model, where firms decide the direction of technological progress so as to maximize their profit. We will show that the log-linear technology condition (20) is endogenously satisfied on the BGP. To keep the analysis from becoming overly complicated, we base this section on a streamlined version of the model of tasks by Irmen (2017) and Irmen and Tabaković (2017) and expand it to incorporate three production factors. There are two benefits from our specification. First, we can analyze intentional R\&Ds within a perfectly competitive economy, which fills a gap between the standard neoclassical growth model (perfectly competitive) and the standard endogenous growth theory (usually with some imperfect competition). Second, while standard endogenous growth models suffer from the scale effects, our model of tasks will become scale independent, which means that we can explain the BGP even when the amount of labor is changing.

\subsection{The Economic Environment}

There are non-overlapping generations of representative firms, each of them existing for only one period. A representative firm performs two types of tasks, M-tasks and N-tasks. The number of M-tasks, as well as that of N-tasks, determines the amount of final output. The M-tasks require effective capital $A_{K, t} K_{t}$ and effective land $A_{X, t} X_{t}$ as inputs, where $A_{K, t}$ and $A_{X, t}$ are the representative firm's capital-augmenting and labor-augmenting technologies (these will be explained in detail below). Effective capital and effective land are substitutable with each other with unit elasticity, which means when more land is used, less capital is required to complete the same task. Specifically, if an M-task uses $A_{X, t} x$ units of effective land, it requires at least $A_{K, t} k \geq\left(A_{X, t} x\right)^{-\zeta}$, $\zeta>0$, units of effective capital. Then, when the representative firm uses $K_{t}$ units of capital and $X_{t}$ unites of land in total, the maximum number of M-tasks it can complete is given by ${ }^{29}$

$$
M_{t}=\left(A_{K, t} K_{t}\right)^{\alpha}\left(A_{X, t} X_{t}\right)^{1-\alpha}, \quad \alpha=1 /(1+\zeta) \in(0,1) .
$$

N-task uses only effective labor, $A_{L, t} L_{t}$, where $A_{L, t}$ is the labor-augmenting technology of the representative firm. Assuming that an N-task requires at least one unit of effective labor, the

\footnotetext{
${ }^{29}$ With unit elasticity of substitution between capital and land, it is optimal to allocate capital and land to individual M-tasks with equal quantities. If the representative firm is to operate $M_{t}$ kinds of M-tasks, it means $k=K_{t} / M_{t}$ and $x=X_{t} / M_{t}$. Substituting these into the input requirement $A_{K, t} k \geq\left(A_{X, t} x\right)^{-\zeta}$ gives $M_{t} \leq\left(A_{K, t} K_{t}\right)^{\alpha}\left(A_{X, t} X_{t}\right)^{1-\alpha}$, where $\alpha=1 /(1+\zeta)$.
} 
maximum number of $\mathrm{N}$-tasks that the representative firm can perform using $L_{t}$ is simply

$$
N_{t}=A_{L, t} L_{t}
$$

By performing $M_{t}$ and $N_{t}$ tasks, the representative firm produces

$$
Y_{t}=\widehat{F}\left(M_{t}, N_{t}\right)=\widehat{F}\left(\left(A_{K, t} K_{t}\right)^{\alpha}\left(A_{X, t} X_{t}\right)^{1-\alpha}, A_{L, t} L_{t}\right)
$$

units of output, where $=\widehat{F}(\cdot)$ is a standard neoclassical production function that has constant returns to scale and an intensive form that satisfies the Inada conditions.

Now, we explain how the factor-specific technologies $\left\{A_{K, t}, A_{X, t}, A_{L, t}\right\}$ are determined. Technical knowledge can be kept within a firm only for one period, after which there are (potentially incomplete) knowledge spillovers. Thus, any firm can freely use a part of the previous period's average factor-augmenting technologies. In addition, the firm can improve each of factor-augmenting technologies through R\&D. Specifically, in a M-task, the capital augmenting technology for that task is given by

$$
A_{K, t}=A_{K, t-1}\left(\underline{\gamma}_{K}+\Phi_{K}\left(i_{k}\right)\right), \quad \underline{\gamma}_{K} \in[0,1],
$$

where we omit the subscript for each task because all M-tasks are symmetric. Here, $\underline{\gamma}_{K}$ is the fraction of the previous period's firms knowledge that is freely available to a period-t firm. Also, $\Phi_{K}\left(i_{k}\right)$ represents the addition of technological knowledge that results investing $i_{k}$ units of final goods in R\&D. Function $\Phi_{K}(\cdot)$ satisfies Inada-like conditions: $\Phi_{K}(0)=0, \Phi_{K}^{\prime}(0)=\infty, \Phi_{K}^{\prime}\left(i_{k}\right)>0$ and $\Phi_{K}^{\prime \prime}\left(i_{k}\right)<0$ for $i_{k}>0$. We also assume that $\Phi_{K}(\infty)>1-\underline{\gamma}_{K}$, which ensures that technology can be improved over time with sufficient amount of R\&D inputs. ${ }^{30}$

It is convenient to rewrite (24) in terms of investment cost function. Let $\gamma_{K, t} \equiv A_{K, t} / A_{K, t-1}$ denote the growth factor of $A_{K, t}$ and $\bar{\gamma}_{K} \equiv \underline{\gamma}_{K}+\Phi_{K}(\infty)$ be its upper bound, which can be either infinity or a finite number. Then we can define the $R \& D$ cost function $\operatorname{as}^{31}$

$$
i_{k}\left(\gamma_{K, t}\right) \equiv \Phi_{K}^{(-1)}\left(\gamma_{K, t}-\underline{\gamma}_{K}\right), \quad \text { defined for } \gamma_{K, t} \in\left[\underline{\gamma}_{K}, \bar{\gamma}_{K}\right]
$$

where $\Phi_{K}^{(-1)}(\cdot)$ denotes the inverse function of $\Phi_{K}(\cdot)$. From properties of $\Phi_{K}(\cdot)$, it can be seen that R\&D cost function $i_{K}\left(\gamma_{K}\right)$ satisfies $i_{K}\left(\underline{\gamma}_{K}\right)=0, i_{K}^{\prime}\left(\underline{\gamma}_{K}\right)=0, i_{K}\left(\bar{\gamma}_{K}\right)=\infty, i_{K}\left(\gamma_{K}\right)>0$, $i_{K}^{\prime}\left(\gamma_{K}\right)>0$, and $i_{K}^{\prime \prime}\left(\gamma_{K}\right)>0$ for all $\gamma_{K} \in\left(\underline{\gamma}_{K}, \bar{\gamma}_{K}\right)$. The marginal cost of improving the technology is small when the innovation is small $\left(\gamma_{K, t} \simeq \underline{\gamma}_{K}<1\right)$, but it becomes increasingly expensive when aiming for bigger innovations, especially approaching the upper bound $\bar{\gamma}_{K}>1 .{ }^{32}$

\footnotetext{
${ }^{30}$ To minimize notations, $\lim _{i_{k} \rightarrow \infty} \Phi_{K}\left(i_{k}\right)$ is written as $\Phi_{K}(\infty)$. We use similar conventions hereafter when they cause no ambiguities.

${ }^{31}$ With a slight abuse of notation, hereafter $i_{K}(\cdot)$ represents a function, not a variable.

${ }^{32}$ This can be explained by congestion in R\&D activities. When many researchers are devoted to improvements
} 
We assume that the firm faces similar constraints when improving $A_{X, t}$ and $A_{L, t} . \mathrm{R} \& \mathrm{D}$ cost functions for land and labor-augmenting technologies are defined accordingly as $i_{X}(\cdot)$ and $i_{L}(\cdot)$, along with $\underline{\gamma}_{X}, \bar{\gamma}_{X}, \underline{\gamma}_{L}$ and $\bar{\gamma}_{L}$, which can differ from $\underline{\gamma}_{K}$ and $\bar{\gamma}_{K}$. Recall that factor augmenting technologies are all task-specific and the R\&D costs must be incurred for each of $M_{t}$ and $N_{t}$ tasks. This means the total R\&D costs for all M- and N-tasks are:

$$
\begin{aligned}
R_{K, t} & =M_{t} \cdot i_{K}\left(A_{K, t} / A_{K, t-1}\right), \\
R_{X, t} & =M_{t} \cdot i_{X}\left(A_{X, t} / A_{X, t-1}\right), \\
R_{L, t} & =N_{t} \cdot i_{L}\left(A_{L, t} / A_{L, t-1}\right) .
\end{aligned}
$$

The objective of the representative firm is to maximize the single period profit, because it lives only for one period and its knowledge will become public next period. By taking the output in each period as numéraire, the period profit is given by

$$
\pi_{t}=\widehat{F}\left(M_{t}, N_{t}\right)-R_{K, t}-R_{X, t}-R_{L, t}-r_{t} K_{t}-\tau_{t} X_{t}-w_{t} L_{t}
$$

where $r_{t}, \tau_{t}$, and $w_{t}$ are interest rate, land rent, and wage rate, respectively.

We keep the demand side of the economy as standard as possible. The economy is populated by a representative households. The size of the representative household (i.e., population) evolves according to

$$
L_{t}=L_{0} g_{L}^{t}, L_{0}>0, g_{L}>0: \text { given }
$$

As in the Ramsey-Cass-Koopman model, the period utility of the household is given by the product of the number of household members and the per capita period felicity function: ${ }^{33}$

$$
u_{t}=L_{t} u\left(C_{t} / L_{t}\right)=L_{0} g_{L}^{t} u\left(C_{t} / L_{0} g_{L}^{t}\right)
$$

where $C_{t} / L_{t}$ is per capita consumption. Following the standard in the literature, we assume the felicity function takes the CRRA form. Then, from (28) and (29), the intertemporal objective function of the household can be written as

$$
U=\sum_{t=0}^{\infty} L_{0}\left(\beta g_{L}\right)^{t} \frac{\left(C_{t} / L_{0} g_{L}^{t}\right)^{1-\theta}-1}{1-\theta},
$$

where $\theta>0$ is the degree of the relative risk aversion and $\beta \in\left(0,1 / g_{L}\right)$ is the discount factor. ${ }^{34}$

in the same task for a given time period, some of them will end up inventing the same innovation. The risk of duplication become more prominent as the input to $\mathrm{R} \& \mathrm{D}$ increases, which makes the $\mathrm{R} \& \mathrm{D}$ cost function $i_{K}(\cdot)$ convex (or, equivalently, the R\&D output function $\Phi_{K}(\cdot)$ concave). See Horii and Iwaisako (2007) for a simple micro foundation.

${ }^{33}$ Strictly speaking, we are using a discrete time version of the standard setting. An equivalent formulation in continuous time can be found, for example, in Chapter 2 of Barro and Sala-i Martin (2004).

${ }^{34}$ We assume $\beta<1 / g_{L}$, or equivalently $\beta g_{L}<1$, so that the household put smaller weights for future generations 
The representative household owns capital, $K_{t}$, and land, $X_{t}$, in addition to labor $L_{t}$. The household also own the representative firm and receives the profit $\pi_{t}$, although in equilibrium we will show that it becomes zero due to perfect competition. For simplicity, we assume that the supply of land is exogenous:

$$
X_{t}=X_{0} g_{X}^{t}, X_{0}, g_{X}>0: \text { given. }
$$

As in the case of population, the available quantity of land can be either constant $g_{X}=1$, shrinking $g_{X} \in(0,1)$, or growing $g_{X}>1 .{ }^{35}$ Physical capital accumulates through the savings of the household.

$$
K_{t+1}=\left(r_{t}+1-\delta\right) K_{t}+\tau_{t} X_{t}+w_{t} L_{t}+\pi_{t}-C_{t}, \quad K_{0}>0: \text { given, }
$$

where $\left(r_{t}+1-\delta\right) K_{t}+\tau_{t} X_{t}+w_{t} L_{t}+\pi_{t}$ represents the household's income. The household is subject to the non-Ponzi game condition:

$$
\lim _{T \rightarrow \infty}\left(\prod_{t=0}^{T}\left(r_{t}+1-\delta\right)\right)^{-1} K_{T+1} \geq 0
$$

This completes the description of the model economy.

Before proceeding to the analysis of the model, let us make sure that it conforms to our definition of the multi-factor neoclassical growth model, given by (1) and (2) in Definition 1. First, the aggregate production function (23) has exactly the same form as (19), which belongs to the definition of the multi-factor neoclassical production function (1). In fact, Proposition 3 guarantees that, if the elasticity of substitution between $K_{t}$ and $X_{t}$ is unity and the economy has a BGP in equilibrium, then the aggregate production function can always be written in the form of (23) at least along the BGP. Our microeconomic setting gives an example of such an economy. Second, by substituting (27) into (32), we obtain the evolution of capital in the same form as (2), where the total R\&D expenditure is defined as $R_{t}=R_{K, t}+R_{X, t}+R_{L, t}$. The difference between Definition 1 and the current model is that we now have a complete description of the economy, including how the speeds of technological changes are determined. We are now ready to explore whether this economy can generate a BGP in equilibrium, paying special attention to whether there is a BGP with strictly positive rate of capital-augmenting technological progress.

\subsection{R\&D by Firms and the Direction of Technological Progress}

Let us examine the behavior of the representative firm in the economy described above, focusing on the role of $R \& D$. The representative firm maximizes profit (27) subject to the production

than the current generation.

${ }^{35}$ Recall that we just call the third production factor $X_{t}$ land for convenience. If $X_{t}$ literally means the acreage of land, then $g_{X}>1$ should be ruled out unless we want to consider space migration. 
and R\&D functions (21)-(26) with respect to $\left\{K_{t}, X_{t}, L_{t}, A_{K, t}, A_{X, t}, A_{L, t}\right\}$, taking as given $\left\{r_{t}\right.$, $\left.\tau_{t}, w_{t}, A_{K, t-1}, A_{X, t-1}, A_{L, t-1}\right\}$. For convenience, let us define by $\mu_{t} \equiv M_{t} / N_{t}$ as the relative task intensity in final good production. Then, because $\widehat{F}(\cdot)$ in (23) is a CRS function, we can write $\widehat{F}\left(M_{t}, N_{t}\right)=N_{t} \widehat{F}\left(\mu_{t}, 1\right) \equiv N_{t} f\left(\mu_{t}\right), \widehat{F}_{M}\left(M_{t}, N_{t}\right)=f^{\prime}\left(\mu_{t}\right)$, and $\widehat{F}_{N}\left(M_{t}, N_{t}\right)=f\left(\mu_{t}\right)-\mu_{t} f^{\prime}\left(\mu_{t}\right) .{ }^{36}$

Using this notation, we can conveniently express the first order conditions for factor demands. The firm demands capital so as to satisfy

$$
r_{t}=\left(\alpha M_{t} / K_{t}\right)\left(f^{\prime}\left(\mu_{t}\right)-i_{K}\left(\gamma_{K, t}\right)-i_{X}\left(\gamma_{X, t}\right)\right)
$$

The RHS of (34) represents the (net) marginal product of $K_{t}$ in producing output $Y_{t}$. It is given by the product of two parts. The first part, $\alpha M / K$, is the marginal product of $K_{t}$ in increasing the number of M-tasks performed in the firm, while the second part is the net marginal product of $M_{t}$ in producing the final output. Note that, in the second part, the innovation cost for an M-task, $i_{K}\left(\gamma_{K, t}\right)+i_{X}\left(\gamma_{X, t}\right)$, is subtracted from the marginal product of $M_{t}, f^{\prime}\left(\mu_{t}\right)$. This happens because when the firm operates more M-tasks, it needs to pay R\&D costs to increase $A_{K, t}$ and $A_{X, t}$ in these tasks so as to keep up with other M-tasks. ${ }^{37}$ The demand for land $X$ is determined in a similar way,

$$
\tau_{t}=\left((1-\alpha) M_{t} / X_{t}\right)\left(f^{\prime}\left(\mu_{t}\right)-i_{K}\left(\gamma_{K, t}\right)-i_{X}\left(\gamma_{X, t}\right)\right)
$$

where $(1-\alpha) M / X$ is the marginal product of $X_{t}$ in performing more M-tasks. Lastly, the firm employs labor according to

$$
w_{t}=A_{L, t}\left(f\left(\mu_{t}\right)-\mu_{t} f^{\prime}\left(\mu_{t}\right)-i_{L}\left(\gamma_{L, t}\right)\right)
$$

where the first part says that an additional unit of $L_{t}$ can perform $A_{L} \mathrm{~N}$-tasks. By substituting (34), (35), and (36) into (27), it can be confirmed that the firm achieves zero profit, $\pi_{t}=0$. This is due to the constant-returns-to-scale property of the firm's problem.

Now, let us turn to R\&D. We first explain the R\&D condition for improving the laboraugmenting technology $A_{L, t}$. The representative firm chooses $A_{L, t}$, or equivalently the speed of technological progress $\gamma_{L, t} \equiv A_{L, t} / A_{L, t-1} \in\left[\underline{\gamma}_{L}, \bar{\gamma}_{L}\right]$, according to first order condition $\partial \pi_{t} / \partial A_{L, t}=0$. Simplifying this condition yields:

$$
\text { R\&D for N-tasks: } \gamma_{L, t} i_{L}^{\prime}\left(\gamma_{L, t}\right)+i_{L}\left(\gamma_{L, t}\right)=f\left(\mu_{t}\right)-\mu_{t} f^{\prime}\left(\mu_{t}\right) .
$$

\footnotetext{
${ }^{36} \widehat{F}_{M}(\cdot)$ and $\widehat{F}_{N}(\cdot)$ represent the partial derivative of function $\widehat{F}(\cdot)$ with respect its first and second arguments, respectively.

${ }^{37}$ Recall that, although we omit subscripts for individual tasks, $A_{K, t}$ and $A_{X, t}$ are task-specific and therefore the $R \& D$ cost will increase with the number of tasks, given the target rate of technological improvement. From the symmetry of tasks within each group ( $\mathrm{M}$ or $\mathrm{N}$ ), it is always optimal to spend equal amounts of R\&D costs for individual tasks.
} 
The firm's private benefit from improving technology $A_{L, t}$ is to become able to perform a larger number of N-tasks, which increases the final output $Y_{t}=\widehat{F}\left(M_{t}, N_{t}\right)$. The RHS of (37) shows the marginal benefit, $\widehat{F}_{N}\left(M_{t}, N_{t}\right)=f\left(\mu_{t}\right)-\mu_{t} f^{\prime}\left(\mu_{t}\right)$. The LHS corresponds to the marginal cost of performing a larger number of N-tasks through augmenting labor efficiency $A_{L, t}$ (given labor employment $L_{t}$ ). This can be achieved by two steps. First, by intensifying the R\&D efforts in the existing N-tasks to raise labor efficiency, the representative firm can save a certain amount of labor, which is just enough to perform one additional N-task. The cost associated with this activity is given by the first term $\gamma_{L, t} i_{L}^{\prime}\left(\gamma_{L, t}\right)$, which we call the intensive marginal $\mathrm{R} \& \mathrm{D}$ cost. The saved labor is then used to perform a new N-task, which means the representative firm needs to invest in R\&D for one more N-task, which costs $i_{L}\left(\gamma_{L, t}\right)$. This extensive marginal R\&D cost is represented by the second term in the LHS.

It is easy to see that condition (37) has a unique solution for $\gamma_{L, t}$ as a function of $\mu_{t}=M_{t} / N_{t}$. From the property of the R\&D cost function, ${ }^{38}$ the LHS of (37), which represents the marginal cost of improving $A_{L}$ so as to operate one additional N-task, is a strictly increasing function of $\gamma_{L, t}=A_{L, t} / A_{L, t-1}$. It takes a value of 0 when $\gamma_{L, t}=1$ and goes to infinity as $\gamma_{L, t} \rightarrow \infty$. ${ }^{39}$ The RHS is the marginal product of task-N, $\widehat{F}_{N}\left(M_{t}, N_{t}\right)$, which is a strictly positive and decreasing function of $\mu_{t}=M_{t} / N_{t}$. Therefore, there always exists a unique interior solution for $\gamma_{L, t}$, which we denote by $\widehat{\gamma}_{L}\left(\mu_{t}\right)$. Note that $\widehat{\gamma}_{L}\left(\mu_{t}\right)>1$ and $\widehat{\gamma}_{L}^{\prime}\left(\mu_{t}\right)>0$ holds for any $\mu_{t}>0$. Intuitively, when the firm is performing relatively fewer N-tasks (i.e., when $\mu_{t} \equiv M_{t} / N_{t}$ is higher), the benefit of increasing $A_{L, t}$ to operate another $\mathrm{N}$-task is larger, and therefore it is optimal to improve the labor-saving technology $A_{L, t}$ at a faster pace $\left(\widehat{\gamma}_{L}\left(\mu_{t}\right)\right.$ should be higher).

Next, we examine the R\&D investments for capital- and land-augmenting technologies. Similarly to the case of labor-augmenting technology, $\gamma_{K, t} \equiv A_{K, t} / A_{K, t-1}$ and $\gamma_{X, t}=A_{X, t} / A_{X, t-1}$ need to satisfy the first order conditions, $\partial \pi_{t} / \partial A_{K, t}=0$ and $\partial \pi_{t} / \partial A_{X, t}=0$. Combining these two equations, we obtain two intuitive conditions that determine the allocation of R\&D efforts among capital- and land-augmenting technologies, as well as the condition that specifies the optimal combined amount of R\&D for M-tasks. ${ }^{40}$

$$
\begin{aligned}
& \text { R\&D allocation: } \frac{\gamma_{K, t} i_{K}^{\prime}\left(\gamma_{K, t}\right)}{\gamma_{X, t} i_{X}^{\prime}\left(\gamma_{X, t}\right)}=\frac{\alpha}{1-\alpha}, \\
& \text { Combined R\&D: }\left(\gamma_{K, t} i_{K}^{\prime}\left(\gamma_{K, t}\right)+i_{K}\left(\gamma_{K, t}\right)\right)+\left(\gamma_{X, t} i_{X}^{\prime}\left(\gamma_{X, t}\right)+i_{X}\left(\gamma_{X, t}\right)\right)=f^{\prime}\left(\mu_{t}\right) .
\end{aligned}
$$

\footnotetext{
${ }^{38}$ Recall that the $\mathrm{R} \& \mathrm{D}$ cost function satisfies $i_{L}(1)=0, i_{L}^{\prime}(1)=0, i_{L}\left(\gamma_{L}\right)>0, i_{L}(\infty)=\infty, i_{L}^{\prime}\left(\gamma_{L}\right)>0$, and $i_{L}^{\prime \prime}\left(\gamma_{L}\right)>0$ for all $\gamma_{L} \geq 1$. The same holds for $i_{K}(\cdot)$ and $i_{X}(\cdot)$.

${ }^{39}$ The derivative of the LHS of (37) with respect to $\gamma_{L, t}$ is $\gamma_{L, t} i_{L}^{\prime \prime}\left(\gamma_{L, t}\right)+2 i_{L}^{\prime}\left(\gamma_{L, t}\right)$, which is strictly positive for all $\gamma_{L, t} \geq 1$ from $i_{L}\left(\gamma_{L, t}\right)>0$ and $i_{L}^{\prime}\left(\gamma_{L, t}\right)>0$. When $\gamma_{L, t}=1$, the LHS becomes $i_{L}^{\prime}(1)+i_{L}(1)=0$. As $\gamma_{L, t} \rightarrow \infty$, the LHS also goes to infinity because $i_{L}(\infty)=\infty$ and $\gamma_{L, t} i_{L}^{\prime}\left(\gamma_{L, t}\right)>0$.

${ }^{40}$ The first order condition for $A_{K, t}$ yields $\left(\gamma_{K, t} / \alpha\right) i_{K}^{\prime}\left(\gamma_{K, t}\right)+i_{K}\left(\gamma_{K, t}\right)+i_{X}\left(\gamma_{X, t}\right)=f^{\prime}\left(\mu_{t}\right)$, whereas that for $A_{X, t}$ gives $\left(\gamma_{X, t} / 1-\alpha\right) i_{X}^{\prime}\left(\gamma_{X, t}\right)+i_{K}\left(\gamma_{K, t}\right)+i_{X}\left(\gamma_{X, t}\right)=f^{\prime}\left(\mu_{t}\right)$. Condition (38) is obtained by subtracting the second equation from the first. Condition (39) is from adding $\alpha$ times the first equation and $(1-\alpha)$ times the second equation.
} 
Observe that $\gamma_{K, t} i_{K}^{\prime}\left(\gamma_{K, t}\right)$ and $\gamma_{X, t} i_{X}^{\prime}\left(\gamma_{X, t}\right)$ in condition (38) have the form similar to the intensive marginal R\&D cost in (37). Since both of them are respectively strictly increasing in $\gamma_{K_{t}}$ and $\gamma_{X, t}$, this condition can be expressed as an upward sloping curve in the $\left(\gamma_{K, t}, \gamma_{X, t}\right)$ space, originating from point $(1,1)$. This condition states that the allocation of R\&D activities for improving capitalaugmenting technology $A_{K, t}$ and labor-augmenting technology $A_{X, t}$ must be reasonably balanced, because the $\mathrm{R} \& \mathrm{D}$ cost function is convex. However, it does not say that $A_{K, t}$ and $A_{X, t}$ must grow at the same speed. As the RHS of condition (39) shows, the allocation should depend on the relative contribution of capital and land in performing M-tasks. When capital's relative contribution is higher (i.e., when $\alpha$ is higher), more resources should be allocated to R\&D for the capitalaugmenting technology. In addition, the slope and convexity of the R\&D cost function also affect the optimal allocation. For example, if it is relatively difficult to improve the land productivity, i.e., if the marginal $\mathrm{R} \& \mathrm{D}$ cost $i_{X}^{\prime}\left(\gamma_{X, t}\right)$ increases more rapidly with its argument than $i_{K}^{\prime}\left(\gamma_{K, t}\right)$, then it is optimal to improve $A_{X, t}$ slower than $A_{K, t}$.

Given allocation rule (38), condition (39) specifies the optimal combined size of R\&D investments. Capital and land are used in M-tasks, and therefore improving capital- and land-augmenting technologies will enable the firm to operate more M-tasks. This marginal benefit is represented by the RHS of $(39), f^{\prime}\left(\mu_{t}\right)=\widehat{F}_{M}\left(M_{t}, N_{t}\right)$. The LHS is the marginal cost of R\&D, which has two parts, $\gamma_{K, t} i_{K}^{\prime}\left(\gamma_{K, t}\right)+i_{K}\left(\gamma_{K, t}\right)$ and $\gamma_{X, t} i_{X}^{\prime}\left(\gamma_{X, t}\right)+i_{X}\left(\gamma_{X, t}\right)$, because both capital- and landaugmenting technologies are to receive some $\mathrm{R} \& \mathrm{D}$ subject to the allocation condition (38). In each of the two parts, the first term represents the intensive marginal $R \& D$ cost, whereas the second term is the extensive marginal R\&D cost, similarly to condition (37).

Note that the first part of the LHS of (39) is increasing in $\gamma_{K, t}$, whereas the second term is in $\gamma_{X, t}{ }^{41}$ Therefore, as shown in Figure 2 the locus of $\left(\gamma_{K, t}, \gamma_{X, t}\right)$ that satisfies the combined R\&D condition (39) is downward sloping on the $\left(\gamma_{K}, \gamma_{X}\right)$ plane. The intersection of the R\&D allocation condition and the combined $\mathrm{R} \& \mathrm{D}$ condition gives the optimal rates of innovation for capital- and land-augmenting technologies. We denote the intersection by $\left(\widehat{\gamma}_{K}\left(\mu_{t}\right), \widehat{\gamma}_{X}\left(\mu_{t}\right)\right)$, which uniquely exists for any $\mu_{t}>0$. When $\mu_{t}$ gets higher, the RHS of (39) declines, which pushes the combined R\&D condition towards the origin. This means that both $\widehat{\gamma}_{K}\left(\mu_{t}\right)$ and $\widehat{\gamma}_{X}\left(\mu_{t}\right)$ are decreasing in $\mu_{t}$. Intuitively, when $M_{t} / N_{t}$ is higher, there is an abundance of M-tasks relative to N-tasks, which reduces the incentives to further improve the M-task related technologies. The following lemma summarizes the results from this subsection.

Proposition 5. (Direction of Technological Change) The representative firm chooses the growth factor of each factor-augmenting technology according to (37), (38) and (39). For any given relative task intensity $\mu_{t} \equiv M_{t} / N_{t}>0$, the solution to this system of equations uniquely exists, and given by $\widehat{\gamma}_{K}\left(\mu_{t}\right), \widehat{\gamma}_{X}\left(\mu_{t}\right)$, and $\widehat{\gamma}_{L}\left(\mu_{t}\right)$. In addition, $\widehat{\gamma}_{K}\left(\mu_{t}\right)<0$ and $\widehat{\gamma}_{X}\left(\mu_{t}\right)<0$ are decreasing

\footnotetext{
${ }^{41}$ This can be confirmed in the same way as in footnote 39
} 


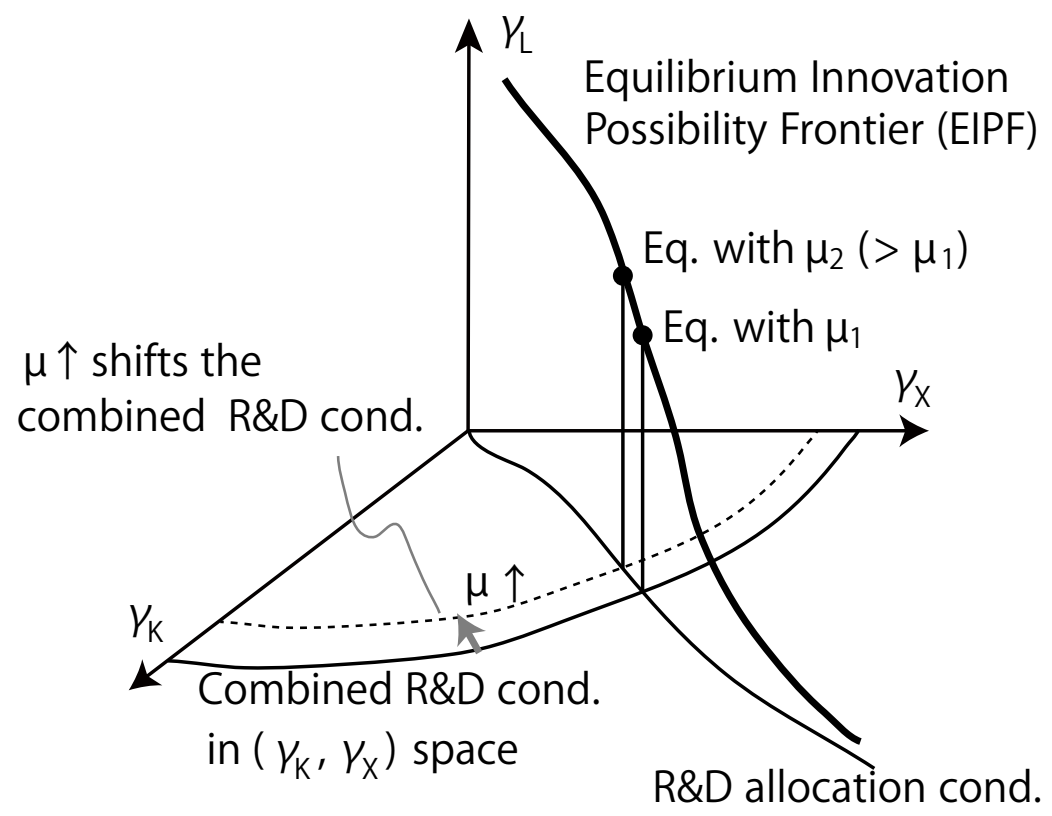

Figure 2: Determination of the direction of the technological change. When $\mu_{t}$ increases, the combined R\&D curve shifts towards the origin, resulting in lower $\gamma_{K}$ and $\gamma_{X}$ and higher $\gamma_{L}$.

continuous functions of $\mu_{t}$, with $\widehat{\gamma}_{K}(0)=\bar{\gamma}_{K}>1, \widehat{\gamma}_{X}(0)=\bar{\gamma}_{X}>1, \widehat{\gamma}_{K}(\infty)=\underline{\gamma}_{K}<1$, and $\widehat{\gamma}_{X}(\infty)=\underline{\gamma}_{X}<1$. On the contrary, $\widehat{\gamma}_{L}\left(\mu_{t}\right)$ is an increasing continuous function with $\widehat{\gamma}_{L}(0)=\underline{\gamma}_{L}<$ 1 and $\widehat{\gamma}_{K}(\infty)=\bar{\gamma}_{L}>1$.

The equilibrium point in Figure 2 depicts the direction of the technological progress implied by Proposition 5 in the 3 -dimensional space. As $\mu_{t}$ changes, the optimal direction moves along the thick curve, which represents the equilibrium innovation possibility frontier (EIPF). The lemma implies that when $\mu_{t}$ is sufficiently low, the firm chooses a strictly positive rate of capital-augmenting technological progress $\left(\widehat{\gamma}_{K}\left(\mu_{t}\right)>1\right)$.

Note that $M_{t}$ also represents the amount of capital composite, which combines effective capital and effective land, whereas $N_{t}$ is equal to the amount of effective labor. Therefore, $\mu_{t}$ can be thought to represent the relative scarcity of effective production factors. In this interpretation, Proposition 5 says that the direction of technological progress is chosen so that it enhances effective factors which are relatively in short supply. Observe that there is no market size effect or scale effects in our model, because the rates of innovation depend only on the ratio $\mu=M_{t} / N_{t}$. This result comes from the assumption that the cost of $\mathrm{R} \& \mathrm{D}$ is proportional to the number of tasks. It is consistent with the observation that larger firms spend more on $R \& D$ while they do not necessarily achieve higher productivity improvements.

Another point to note is that $\mu_{t}$ is endogenous and depends on the amount of R\&D activities in period $t$. Therefore, Proposition 5 should be understood as an equilibrium relationship between the task intensity and the direction of technological change, rather than causality. To see how they 
are determined in each period, we need to consider the equilibrium dynamics.

\subsection{Equilibrium Dynamics}

The equilibrium path of this economy is given by the sequence of output, consumption, production factors, technologies, and R\&D investments, $\left\{Y_{t}, C_{t}, K_{t}, X_{t}, L_{t}, A_{K, t}, A_{X, t}, A_{L, t}, R_{K, t}, R_{X, t}, R_{X, t}\right\}_{t=0}^{\infty}$, which satisfy the representative firm's optimization, the representative consumer's utility maximization, and the market clearance for output and production factors. The economy is endowed with $K_{0}, X_{0}$ and $L_{0}$ at time 0 , as well as the initial levels of publicly available technologies, $A_{K,-1}, A_{X,-1}$ and $A_{L,-1}$.

While the equilibrium involves many variables, we can analytically characterize its dynamic path in terms of only three variables: relative task intensity $\mu_{t}=M_{t} / N_{t}$, the amount of capital per effective labor $k_{t} \equiv K_{t} / A_{L, t} L_{t}$, and consumption per effective labor $c_{t} \equiv C_{t} / A_{L, t} L_{t}$. Below, we construct the equilibrium mapping from $\left\{\mu_{t}, k_{t}, c_{t}\right\}$ to $\left\{\mu_{t+1}, k_{t+1}, c_{t+1}\right\}$ for $t \geq 0$. The mapping and the initial conditions $\mu_{0}$ and $k_{0}$, together with the transversality condition for $c_{t}$, will pin down the equilibrium path of $\left\{\mu_{t}, k_{t}, c_{t}\right\}$, from which the path of all variables in the model can be recovered.

Before so doing, it is convenient to define the net aggregate output in the economy as $V_{t}=$ $\widehat{F}\left(M_{t}, N_{t}\right)-R_{K, t}-R_{X, t}-R_{L, t}$, which means the aggregate output minus the total R\&D costs in the economy. The net output per effective labor can be written as a function of $\mu_{t}$ :

$$
V_{t} / N_{t}=f\left(\mu_{t}\right)-\mu_{t}\left(i_{K}\left(\widehat{\gamma}_{K}\left(\mu_{t}\right)\right)+i_{X}\left(\widehat{\gamma}_{X}\left(\mu_{t}\right)\right)\right)-i_{L}\left(\widehat{\gamma}_{L}\left(\mu_{t}\right)\right) \equiv v\left(\mu_{t}\right)
$$

Then, substituting the profit (27) into the budget constraint (32), we can express the growth of aggregate capital supply in terms of $\mu_{t}, k_{t}$ and $c_{t}$ :

$$
\frac{K_{t+1}}{K_{t}}=\frac{V_{t}+(1-\delta) K_{t}-C_{t}}{K_{t}}=\frac{v\left(\mu_{t}\right)-c_{t}}{k_{t}}+1-\delta .
$$

Dynamics for $\mu_{\mathbf{t + 1}}$. The growth factor of $\mu_{t+1}$ is defined by $\mu_{t+1} / \mu_{t}=\left(M_{t+1} / M_{t}\right) /\left(N_{t+1} / N_{t}\right)$. By using (21), (22), (28), (31) and (41), its value in equilibrium can be written as

$$
\frac{\mu_{t+1}}{\mu_{t}}=\frac{\left(g_{X} \widehat{\gamma}_{X}\left(\mu_{t+1}\right)\right)^{1-\alpha}}{g_{L} \widehat{\gamma}_{L}\left(\mu_{t+1}\right)}\left(\widehat{\gamma}_{K}\left(\mu_{t+1}\right)\left(\frac{v\left(\mu_{t}\right)-c_{t}}{k_{t}}+1-\delta\right)\right)^{\alpha},
$$

where $\widehat{\gamma}_{K}\left(\mu_{t}\right), \widehat{\gamma}_{X}\left(\mu_{t}\right)$, and $\widehat{\gamma}_{L}\left(\mu_{t}\right)$ are the rates of technological innovations defined in Proposition 5. While equation (42) gives a relationship between the period-t variables $\left\{\mu_{t}, k_{t}, c_{t}\right\}$ and the next period's $\mu_{t+1}$, it is not easy to understand how $\mu_{t+1}$ is determined since both the LHS and the RHS depends on $\mu_{t+1}$.

To interpret it intuitively, let us decompose the dynamic relationship in (42) into two steps. 
First, we define the pre-R\&D relative factor intensity by

$$
\mu_{t+1}^{\mathrm{pre}} \equiv \frac{\left(A_{K, t} K_{t+1}\right)^{\alpha}\left(A_{X, t} X_{t+1}\right)^{1-\alpha}}{A_{L, t} L_{t+1}}=\frac{g_{X}^{1-\alpha}}{g_{L}}\left(\frac{v\left(\mu_{t}\right)-c_{t}}{k_{t}}+1-\delta\right)^{\alpha} \mu_{t}
$$

where the last equality is from (28), (31), (41) and the definition of $\mu_{t}$. It is the value of $\mu_{t+1}$ before technologies are improved from their period- $t$ state. Second, $\mu_{t+1}^{\text {pre }}$ and the post-R\&D value of $\mu_{t+1}$ is related by the growth of technological levels $\widehat{\gamma}_{K}\left(\mu_{t}\right), \widehat{\gamma}_{X}\left(\mu_{t}\right)$, and $\widehat{\gamma}_{L}\left(\mu_{t}\right)$ as follows:

$$
\mu_{t+1}^{\mathrm{pre}}=\frac{\widehat{\gamma}_{L}\left(\mu_{t+1}\right)}{\widehat{\gamma}_{K}\left(\mu_{t+1}\right)^{\alpha} \widehat{\gamma}_{X}\left(\mu_{t+1}\right)^{1-\alpha}} \mu_{t+1} \equiv \Gamma\left(\mu_{t+1}\right)
$$

Note that, Proposition 5 implies that function $\Gamma\left(\mu_{t+1}\right)$ is a strictly increasing function with $\lim _{\mu \rightarrow 0} \Gamma(\mu)=$ 0 and $\lim _{\mu \rightarrow \infty} \Gamma(\mu)=\infty$. Therefore, its inverse function $\mu_{t+1}=\Gamma^{(-1)}\left(\mu_{t+1}^{\mathrm{pre}}\right)$ is well-defined for all $\mu_{t+1}^{\mathrm{pre}}>0$, and is a strictly increasing function.

Using this inverse function and (43), the dynamic relationship (42) can be written as

$$
\mu_{t+1}=\Gamma^{(-1)}\left(\frac{g_{X}^{1-\alpha}}{g_{L}}\left(\frac{v\left(\mu_{t}\right)-c_{t}}{k_{t}}+1-\delta\right)^{\alpha} \mu_{t}\right) \equiv \psi^{\mu}\left(\mu_{t}, k_{t}, c_{t}\right)
$$

Equation (45) explains how $\mu_{t+1}$ is determined given the period- $t$ variables $\mu_{t}, k_{t}, c_{t}$. We write this mapping as $\mu_{t+1}=\psi^{\mu}\left(\mu_{t}, k_{t}, c_{t}\right)$. It provides a natural 2-step interpretation of the equivalent equation (42). The argument of function $\Gamma^{(-1)}(\cdot)$ in (45) represent the pre-R\&D relative task intensity, which is determined by the relative supply of production factors, as well as the period- $t$ technology levels. Then, function $\Gamma^{(-1)}(\cdot)$ describes how $\mathrm{R} \& \mathrm{D}$ in period $t+1$ transforms the relative task intensity.

Dynamics for $\mathbf{k}_{\mathbf{t}+\mathbf{1}}$. From (28) and (41), the growth factor of $k_{t} \equiv K_{t} / A_{L, t} L_{t}$ is obtained as

$$
\frac{k_{t+1}}{k_{t}}=\frac{1}{g_{L} \widehat{\gamma}_{L}\left(\mu_{t+1}\right)}\left(\frac{v\left(\mu_{t}\right)-c_{t}}{k_{t}}+1-\delta\right) .
$$

While $\mu_{t+1}$ is present in the RHS, we can replace it with (45) so that the RHS depend only on the variables in period $t$.

$$
k_{t+1}=\frac{1}{g_{L} \widehat{\gamma}_{L}\left(\psi^{\mu}\left(\mu_{t}, k_{t}, c_{t}\right)\right)}\left(v\left(\mu_{t}\right)-c_{t}+(1-\delta) k_{t}\right) \equiv \psi^{k}\left(\mu_{t}, k_{t}, c_{t}\right)
$$

This dynamic equation simply represents the process of capital accumulation per effective labor. The expression $\left(v\left(\mu_{t}\right)-c_{t}+(1-\delta) k_{t}\right)$ shows the sum of the net saving and the undepreciated part of existing capital, per effective labor in period $t$. It must be divided by $g_{L} \widehat{\gamma}_{L}$ because of the growth of effective labor between period $t$ and $t+1$. 
Dynamics for $\mathbf{c}_{\mathbf{t}+\mathbf{1}}$. The representative household maximizes the intertemporal utility function (30) subject to the budget constraint (32) and the non-Ponzi Game condition (33). The Euler equation for this problem is $C_{t}^{-\theta}=\left(r_{t+1}+1-\delta\right) \beta g_{L}^{\theta} C_{t+1}^{-\theta}$. By substituting the market interest rate (34) into the Euler equation and then applying it to the definition $c_{t} \equiv C_{t} / A_{L, t} L_{t}$, we obtain the growth factor of consumption per effective labor:

$$
\frac{c_{t+1}}{c_{t}}=\frac{\beta^{1 / \theta}}{\widehat{\gamma}_{L}\left(\mu_{t+1}\right)}\left(\frac{\alpha \mu_{t+1}}{k_{t+1}}\left(f^{\prime}\left(\mu_{t+1}\right)-i_{K}\left(\widehat{\gamma}_{K}\left(\mu_{t+1}\right)\right)-i_{X}\left(\widehat{\gamma}_{X}\left(\mu_{t+1}\right)\right)\right)+1-\delta\right)^{1 / \theta} .
$$

By replacing the period- $(t+1)$ variables in the RHS by (45) and (47), we can rewrite equation (48) as

$$
\begin{array}{r}
c_{t+1}=\frac{\beta^{1 / \theta} c_{t}}{\widehat{\gamma}_{L}\left(\psi^{\mu}\left(\mu_{t}, k_{t}, c_{t}\right)\right)}\left(\frac { \alpha \psi ^ { \mu } ( \mu _ { t } , k _ { t } , c _ { t } ) } { \psi ^ { k } ( \mu _ { t } , k _ { t } , c _ { t } ) } \left(f^{\prime}\left(\psi^{\mu}\left(\mu_{t}, k_{t}, c_{t}\right)\right)-i_{K}\left(\widehat{\gamma}_{K}\left(\psi^{\mu}\left(\mu_{t}, k_{t}, c_{t}\right)\right)\right)\right.\right. \\
\left.\left.-i_{X}\left(\widehat{\gamma}_{X}\left(\psi^{\mu}\left(\mu_{t}, k_{t}, c_{t}\right)\right)\right)\right)+1-\delta\right)^{1 / \theta} \equiv \psi^{c}\left(\mu_{t}, k_{t}, c_{t}\right) .
\end{array}
$$

Equations (45), (47) and (49) constitute the equilibrium mapping from $\left\{\mu_{t}, k_{t}, c_{t}\right\}$ to $\left\{\mu_{t+1}, k_{t+1}, c_{t+1}\right\}$ for all $t \geq 0$.

Boundary Conditions. To obtain the equilibrium path of $\left\{\mu_{t}, k_{t}, c_{t}\right\}_{t=0}^{\infty}$, we need three boundary conditions. First, since $K_{0}, X_{0}, L_{0}, A_{K,-1}, A_{X,-1}$ and $A_{L,-1}$ are given, we can construct $\mu_{0}^{\text {pre }}$, the pre-R\&D relative task intensity for period 0 . Using it with the inverse function of $\Gamma$ from (44), we have the initial value of $\mu_{t}$ :

$$
\mu_{0}=\Gamma^{(-1)}\left(\frac{\left(A_{K,-1} K_{0}\right)^{\alpha}\left(A_{X,-1} X_{0}\right)^{1-\alpha}}{A_{L,-1} L_{0}}\right) .
$$

Second, using $\mu_{0}$, the initial value of $k_{t}$ is readily obtained by

$$
k_{0}=\frac{K_{0}}{\widehat{\gamma}_{L}\left(\mu_{0}\right) A_{L,-1} L_{0}}
$$

Finally, the initial value of $c_{t}$ must be chosen so as to satisfy the non-Ponzi game condition and the transversality condition, which jointly requires the condition (33) to hold with equality. ${ }^{42}$ By using the Euler equation, the terminal condition can be written as

$$
\lim _{T \rightarrow \infty}\left(\beta g_{L} \widehat{\gamma}_{L}\left(\mu_{T}\right)^{1-\theta}\right)^{T} c_{T}^{-\theta} k_{T}=0
$$

The next subsection will show that the economy has a BGP that satisfies this terminal condition.

\footnotetext{
${ }^{42}$ The transversality condition from the household's maximization problem takes a similar form to (33) but has " $\leq$ " instead of " $\geq$ ".
} 


\subsection{The Balanced Growth Path}

Now we are ready to derive the balanced growth path (BGP) of this economy. It will be shown that the direction of technological progress, including strictly positive capital-augmenting technological progress, is endogenously chosen so that the BGP is achieved in equilibrium. Let us start by defining the BGP in this economy and then showing that the three key variables are constant on it.

Lemma 3. Define a $B G P$ as an equilibrium path where the growth factors of $\left\{Y_{t}, K_{t}, X_{t}, L_{t}, C_{t}\right.$, $\left.R_{t}, M_{t}, N_{t}\right\}$ are all constant. ${ }^{43}$ Then, on any BGP, the values of $\mu_{t}, k_{t}$ and $c_{t}$ must be constant.

Proof. See Appendix Section A.2.

Let us denote the BGP values of $\mu_{t}, k_{t}$ and $c_{t}$ by $\mu^{*}, k^{*}$ and $c^{*}$, respectively. Note that the RHS of (42), (46) and (48) represent the growth factors of $\mu_{t}, k_{t}$ and $c_{t}$, and therefore they take the value of 1 on the BGP. Rearranging them, we obtain the values of $\mu^{*}, k^{*}$ and $c^{*}$ as follows.

First, from (42) and (46), the BGP value of $\mu_{t} \equiv M_{t} / N_{t}$ will satisfy

$$
\left(g_{X} \widehat{\gamma}_{X}\left(\mu^{*}\right)\right)^{1-\alpha}\left(\widehat{\gamma}_{K}\left(\mu^{*}\right)\right)^{\alpha}=\left(g_{L} \widehat{\gamma}_{L}\left(\mu^{*}\right)\right)^{1-\alpha} .
$$

We can confirm that there exist a unique value of $\mu^{*}>0$ that satisfies condition (53). Since $\widehat{\gamma}_{K}^{\prime}\left(\mu^{*}\right)<0, \widehat{\gamma}_{X}^{\prime}\left(\mu^{*}\right)<0$ and $\widehat{\gamma}_{L}^{\prime}\left(\mu^{*}\right)>0$ from Proposition 5, the LHS is strictly downward sloping with respect to $\mu^{*}$, while the RHS is strictly upward sloping. This guarantees the uniqueness of $\mu^{*}$. An intuitive way to interpret (53) is to multiply the both of its sides by $\left(g_{L} \widehat{\gamma}_{L}\left(\mu^{*}\right)\right)^{\alpha}$.

$$
\left(g_{X} \widehat{\gamma}_{X}\left(\mu^{*}\right)\right)^{1-\alpha}\left(\widehat{\gamma}_{K}\left(\mu^{*}\right) g_{L} \widehat{\gamma}_{L}\left(\mu^{*}\right)\right)^{\alpha}=g_{L} \widehat{\gamma}_{L}\left(\mu^{*}\right)=g^{*}
$$

The LHS represents the growth factor of $M_{t}$ on the BGP, while the RHS is that for $N_{t}$. Therefore, this condition means that the relative factor intensity $\mu^{*}=M_{t} / N_{t}$ is determined so that $M_{t}$ and $N_{t}$ grow at the same speed on the Equilibrium Innovation Possibility Frontier (EIPF), as depicted in Figure 2. Because of the CRS property of production function $Y_{t}=\widehat{F}\left(M_{t}, N_{t}\right)$, the value of equation (53) also represents the the economic growth factor $g^{*} \equiv Y_{t+1} / Y_{t}$.

Second, from (48), the BGP value of $k_{t}=K_{t} /\left(A_{t} L_{t}\right)$ is

$$
k^{*}=\frac{\beta \alpha \mu^{*}\left(f^{\prime}\left(\mu^{*}\right)-i_{K}\left(\widehat{\gamma}_{K}\left(\mu^{*}\right)\right)-i_{X}\left(\widehat{\gamma}_{X}\left(\mu^{*}\right)\right)\right)}{\widehat{\gamma}_{L}\left(\mu^{*}\right)^{\theta}-\beta(1-\delta)},
$$

where $\mu^{*}$ is obtained from (53). Intuitively, (55) means that the capital-effective labor ratio on the BGP is determined so that the interest rate $r^{*}$ implies constant consumption per effective labor,

\footnotetext{
${ }^{43}$ Here we slightly extend Definition 2 by requiring constancy of the growth factors of $M_{t}$ and $N_{t}$, i.e., the numbers of tasks performed in the economy.
} 
$c^{*}{ }^{44}$ Third, from the resource constraint (46), the BGP value of $c^{*}=C_{t} / A_{L, t} L_{t}$ must satisfy

$$
c^{*}=v\left(\mu^{*}\right)+(1-\delta) k^{*}-g_{L} \widehat{\gamma}_{L}\left(\mu^{*}\right) k^{*},
$$

where $\mu^{*}$ and $k^{*}$ are given by (53) and (55). These three equations uniquely give the BGP in this economy.

Proposition 6. There exists a unique BGP that satisfy $\mu^{*}>0, k^{*}>0, c^{*}>0$, and the terminal condition (52).

Note that (53) is equivalent to the technology condition (20) in Section 5.3, except that now the speeds of technological progress are endogenously determined. This difference has an important implication for the plausibility of capital-augmenting technological progress on the BGP. In the example model in Section 5.3, the rates of innovation for three factor-augmenting technologies are exogenously given, and they must happen to satisfy a knife-edge condition (20). In contrast, in the full model of this section, the representative firm chooses the rates of technological progress so as to maximize its profit.

This balanced growth path accommodates the two previously-conflicting stylized facts: (i) a lessthan-unitary elasticity of substitution between labor and capital, and (ii) strictly positive capital augmenting technological change in the long run. To demonstrate this result, section 7 explores the model numerically. The primary benefit of the exercise is to show that the endogenous growth model has a locally stable BGP with a positive rate of capital-augmenting technical change. We also show that the model can be readily calibrated to standard macroeconomic facts describing the BGP of the United States.

\section{Numerical Analysis and Stability}

The multi-factor Uzawa steady state growth theorem presented in Proposition 1 and 3 requires that the growth rates of factor-augmenting technologies conform to a particular log-linear relationship as in equation (20). Section 6 demonstrates that this log-linear relationship can be an endogenous outcome of a model with directed technical change, suggesting that this requirement is not actually restrictive. The analytic results, however, did not demonstrate that the economy will necessarily converge to the BGP with capital-augmenting technical change.

In this section, therefore, we investigate the local and global stability of the three factor endogenous growth model. To do so, we present a series of numerical examples for which we can check both local and global stability computationally. Whenever possible, we ensure that our numerical examples are consistent with macroeconomic data characterizing the balanced growth path of the

\footnotetext{
${ }^{44}$ Using (34), condition (55) is shown to be equivalent to the Euler equation on the BGP, $\widehat{\gamma}_{L}\left(\mu^{*}\right)=\beta\left(r^{*}+1-\delta\right)$. The LHS means the growth of consumption per capita (when consumption per effective labor is constant).
} 
United States. We stress, however, that this is not a complete calibration, and the results would be insufficient for a complete quantitative analysis. Our contribution is theoretical, and the numerical results are meant to be informative about stability. As discussed in section 8, future empirical and quantitative work to fully pin down the parameters of such a growth model is a particularly promising area for future work.

\subsection{Calibration}

\subsubsection{Preliminaries}

In this section, we describe the partial calibration procedure that we use to discipline our numerical examples. We start by making some important assumptions. In line with Section 6, we assume that there is some aggregate variable $X$ that incorporates all factors of production other than labor and capital. ${ }^{45}$ For ease of exposition, we call this factor land.

Next, we need to make two important functional form assumptions. We start by assuming that the aggregate production function is CES,

$$
Y_{t}=\left\{\eta\left(\left(A_{K} K\right)^{\alpha}\left(A_{X} X\right)^{1-\alpha}\right)^{\frac{\epsilon-1}{\epsilon}}+(1-\eta)\left(A_{L} L\right)^{\frac{\epsilon-1}{\epsilon}}\right\}^{\frac{\epsilon}{1-\epsilon}}
$$

Next, we assume that the R\&D production function takes the form

$$
\Phi\left(i_{Z}\right)=1+\tilde{\zeta}_{Z} R_{Z}^{1-\tilde{\lambda}}
$$

which implies that $i_{Z}=\zeta_{Z}\left(\gamma_{Z}-1\right)^{\lambda}$, where $\zeta_{Z}=\tilde{\zeta}_{Z}^{-\frac{1}{1-\tilde{\lambda}}}$ and $\lambda=\frac{1}{1-\tilde{\lambda}}$. In words, we assume that the productivity of $R \& D$ investment differs across types of technology due to the $\zeta_{Z}$ parameters, but that all technologies experience the same degree of diminishing returns.

Finally, we need to decide on a period length. In the endogenous growth model, one period captures the length of a patent and, equivalently, the horizon for firm decision-making. We take a period length of 10 years as a reasonable approximation.

\subsubsection{External Calibration}

We set several parameters exogenously based on existing literature. To start, we take $\beta=(.025)^{10}=$ .86 and $\theta=1$ from Golosov et al. (2014), who use an identical period length. We also set the elasticity of substitution between labor and the capital composite exogenously. Existing studies generally estimate this parameter assuming that there are only two factors of production, reproducible capital and labor. Thus, any existing match from the literature to our model is imperfect. We stress again that this is meant to be an illustrative example demonstrating that the stability of the three-factor

\footnotetext{
${ }^{45}$ This is done for transparency and tractability. The theorem only requires that any one factor non-reproducible factor is combined with capital with a unit-elastic elasticity of substitution.
} 
model for a wide range of parameters. For our baseline analysis, we take $\epsilon=0.7$ from Oberfield and Raval (2014), which is similar to estimates by Antras et al. (2004), Klump et al. (2007), Herrendorf et al. (2015) and Alvarez-Cuadrado et al. (2018). Karabarbounis and Neiman (2014) and Piketty (2014) estimate elasticities that are greater than one.

In the next section, we describe how the remaining parameters are calibrated. We are not able to pin down $\lambda$, the degree of diminishing marginal returns in R\&D. Intuitively, $\lambda$ governs the diminishing returns to reallocating research inputs between different types of technology. However, research allocations are stable on the balanced growth path, making it difficult to pin down this parameter. In the baseline, we take $\lambda=2$, so that the cost function is quadratic. We also show that our stability results do not depend on this choice.

\subsubsection{BGP Moments}

To calibrate the remaining parameters, we match the model to balanced growth from the United States. $^{46}$ Average annual growth in GDP per capita in the United States from 1950-2014 was $2.0 \%$ year $\left(\gamma_{L}^{*}=1.22\right)$, population growth was $1.1 \% /$ year $\left(g_{L}=1.12\right)$, and the relative price of investment goods fell by $0.6 \% /$ year $\left(\gamma_{K}^{*}=1.06\right)$. The most obvious interpretation of $X$ is that is a a land-energy composite. Interpreting $X$ as land would suggest that it is fixed over time $\left(g_{X}=1\right)$, which we will take as the baseline.

We use the notation $\kappa_{Z}$ to denote the income share of factor $Z$. The average labor share of income over this period was $\kappa_{L}^{*}=63 \%$, and based on Table 2 we take $\kappa_{X}^{*}=10 \%$, as it is a stand in for all forms of natural capital. This immediately implies that $\kappa_{K}^{*}=27 \%$.

Finally, we match the model to two important macroeconomic aggregates from the U.S. data. The annual capital-output ration in the United States is 3, when measuring capital in terms of final output as in our model. With our ten year time scale, we target a value of 0.3 . We also target a saving rate of $11 \%$, which corresponds to non-R\&D investment in our model.

Noting that we set $\lambda$ exogenously - and test for robustness - the remaining unknown parameters are given by the set $\left\{\zeta_{K}, \zeta_{X}, \zeta_{L}, \eta, \delta\right\}$. To find these parameters, we perform an ad hoc search over the relevant parameter space. In the future, this will be updated to a more formal procedure. The results are presented in Table 2 .

As demonstrated in Table 3, the ad hoc calibration is able to match several key moments from the data. ${ }^{47}$. As noted above, our goal is not to provide a complete quantitative model of the U.S. economy. Still, the fact that the model is able to recreate patterns observed in data suggests that it will be useful as a quantitative tool in future work. ${ }^{48}$

\footnotetext{
${ }^{46}$ See Appendix Section A.1 for details regarding the data used in the calibration.

${ }^{47} \mathrm{~A}$ more formal calibration will be added soon

${ }^{48} \mathrm{In}$ terms of non-targeted moments, it is worth noting that we predict an R\&D share of output of $6.7 \%$ which is higher than the standard measure of R\&D expenditure, which makes up approximately $2.7 \%$ of GDP. This result is not surprising. Given that our model is attributing all changes in productivity to $\mathrm{R} \& \mathrm{D}$, it should incorporate more
} 
Table 2: Calibration

\begin{tabular}{cccc}
\hline \hline Parameter & Baseline & Description & Source \\
\hline$\lambda$ & 2.0 & R\&D Convexity & Free Parameter \\
$g_{X}$ & 1.0 & Growth of $X$ & Definition of $X$ \\
$\epsilon$ & 0.7 & Elast. Sub. & Oberfield and Raval (2014) \\
\hline$\beta$ & 0.78 & Discount factor & Golosov et al. (2014) \\
$\theta$ & 1.00 & IES & Golosov et al. (2014) \\
$g_{L}$ & 1.01 & Population growth & BEA \\
\hline$\delta$ & .25 & Depreciation & Calibrated \\
$\alpha$ & 0.68 & Cap. Distribution & Calibrated \\
$\eta$ & 0.24 & CES Distribution & Calibrated \\
$\zeta_{L}$ & 0.81 & Research efficiency & Calibrated \\
$\zeta_{K}$ & 3.75 & Research efficiency & Calibrated \\
$\zeta_{X}$ & 0.60 & Research efficiency & Calibrated \\
\hline \hline
\end{tabular}

Table 3: Moments

\begin{tabular}{cccc}
\hline \hline Moment & Description & Model & Data \\
\hline$\gamma_{L}^{*}$ & $Y / L$ growth & 1.22 & 1.21 \\
$\gamma_{K}^{*}$ & $A_{K}$ growth & 1.07 & 1.06 \\
$\kappa_{L}^{*}$ & Labor share & 0.61 & 0.63 \\
$\kappa_{X}^{*}$ & Land Share & 0.09 & 0.10 \\
$k / y$ & Capital-Output & 0.23 & 0.30 \\
$s^{*}$ & Savings Rate & 0.14 & 0.11 \\
\hline \hline
\end{tabular}

\subsection{Local Stability}

Next, we turn to turn to examining the stability of the numerical model. As a preliminary analysis, we hold fixed all the value from Table 2, except for $\lambda$. We then solve for the steady state for different values of $\lambda$ and numerically find the eigenvalues of the system. In the future, this process will be updated to test a much wider range of parameters. Based on other initial analyses (not reported), we find evidence that the system is stable for a wide range of parameter values.

Table 4: Stability

\begin{tabular}{ccc}
\hline \hline$\lambda$ & Eigenvalues & Stable? \\
\hline 1.25 & $0.92,0.57,2.26$ & $\mathrm{Y}$ \\
1.5 & $0.91,0.48,2.46$ & $\mathrm{Y}$ \\
2 & $0.92,0.39,2.96$ & $\mathrm{Y}$ \\
3 & $0.94,0.27,4.22$ & $\mathrm{Y}$ \\
4 & $0.95,0.19,6.03$ & $\mathrm{Y}$ \\
\hline \hline
\end{tabular}

Table 4 shows that the numerical models is locally saddle-path stable for all the different types of investment than business investment (e.g., education expenditures). 
parameter values of $\lambda$. Thus, for at least some range of starting positions, the endogenous growth model endogenous converges to a balanced growth path with a positive rate of capital-augmenting technical change. This result demonstrates that the log-linear relationship between technological growth rates can be viewed as an endogenous outcome and does not pose an extra restriction on the conditions needed for balanced growth in neoclassical models with improvements in the productivity of capital.

\section{Discussion}

In this section, we discuss the implications of the model, as well as some important goals for future research.

\subsection{Implications of the endogenous growth model}

The multi-factor Uzawa steady state theorem and the endogenous growth model suggest that natural resources and directed technical change are fundamental to understanding the evolution of the economy (Acemoglu, 1998, 2002). There is, of course, a long literature on both of these topics, but they are generally only included in growth models to achieve specific aims. For example, energy is generally only included in growth models when studying the depletion of finite resources (e.g., Hotelling, 1931; Heal, 1976) or climate change (e.g., Golosov et al., 2014; Barrage, 2019). Directed technical change is often used to understand the evolution of relative wages between different groups of workers (e.g., Acemoglu, 1998, 2002; Acemoglu and Restrepo, 2016).

Our results suggests a much broader importance of directed technical change and natural resources - these factors must be incorporated into models of economic growth in order to recreate the balanced growth facts that originally motivated aggregate growth modelling (Solow, 1956, 1994). This suggests that essentially all analyses in economic growth would benefit from including natural resources and directed technical change if they want to be consistent with balanced growth. We think that our three-factor endogenous growth model presents a natural starting point for such analyses. ${ }^{49}$ In this way, our results are related to those of Unified Growth Theory, which suggests that including land in the production function is essential of understanding long-run economic growth and the transition from Malthusian stagnation to modern growth (e.g., Galor and Weil, 2000; Hansen and Prescott, 2002; Galor, 2011). Also, Caselli and Feyrer (2007) suggest that accounting for natural resource well is necessary for understanding cross-country dispersion in the marginal product of capital.

\footnotetext{
${ }^{49}$ Existing work on directed technical change and the environment may also be helpful in this regard (e.g, Smulders and De Nooij, 2003; Acemoglu et al., 2012; Hassler et al., 2016).
} 


\subsection{Next Steps}

Estimating $\sigma_{K X_{j}}$ - The obvious next step for future research would be to determine whether there is some factor, $X_{J}$, that has a unit-elastic elasticity of substitution with reproducible capital. In our illustrative growth model, we assumed that this third factor was an amalgamation of all factors not included in reproducible capital or labor. Proposition 3, however, only requires that a single factor of production has this property. Thus, it is necessary to test many different factors to see if they satisfy this property. ${ }^{50}$ The obvious candidates would be land, energy, and materials, but it could also be any subcategory of these types of inputs. Given the vast array of choices and the limited restrictions created by the theory, we think it is quite plausible that such a factor exists.

What if there is no $X_{J}$ with $\sigma_{K X_{j}}=1$ ? - Despite the wide range of factors used in production, it is possible that no factor has unit-elastic substitution with reproducible capital. In this case, proposition 3 suggests that it is not possible to write down a multi-factor growth neoclassical growth model with capital-augmenting technical change on the balanced growth path. This is a restrictive condition that is clearly at odds with data. Such a result, therefore, may suggest the need to focus on disaggregated models - or models with constant, rather than balanced, growth (Jones, 2002) in order to understand the causes and consequences of technical change. ${ }^{51}$

Another approach, followed by Grossman et al. (2017), is to develop growth models where there is some force - like schooling - that exactly offsets improvements in capital-augmenting technology. On the balanced growth path, such models are isomorphic to standard models without capitalaugmenting technical change. If factor shares are constant, as in Grossman et al. (2017), they are also isomorphic to models without capital-augmenting technical change near the balanced growth path. Examining the empirical relevance of these assumptions is another important goal for future research aimed at understanding balanced growth.

Applications for a multi-factor model - The Uzawa steady state theorem is not a mere theoretical curiosity. It has important implications for understanding the economy. If it is possible to identify a factors that has a unit-elastic substitution with reproducible capital, then aggregate productions can include both labor- and capital-augmenting technical change on the balanced growth path, allowing for more informative analysis of data and policy interventions.

Most obviously, the counter-factual notion that there is a single type of technology places severe restrictions on our ability to describe the economy, even in an aggregate sense. In growth accounting, there has been a great deal of work focusing on the role of investment specific technical

\footnotetext{
${ }^{50}$ While, of course, avoiding the pitfalls of multiple hypothesis testing.

${ }^{51}$ This result would be consistent with other recent work arguing that the conditions for balanced growth in endogenous growth models are not satisfied in the data (e.g., Jones, 2002; Bloom et al., 2017). See Growiec (2007) for a general discussion of knife-edge conditions required to have balanced growth in aggregate models.
} 
change. To conform with the restrictions of the Uzawa theorem, the production functions in such analyses need must focus on the Cobb-Douglas case (e.g., Greenwood et al., 1997; Krusell, 1998; Cummins and Violante, 2002) or be inconsistent with balanced growth (e.g., Krusell et al., 2000). ${ }^{52}$ Incorporating additional factors of production would allow such exercises to be conducted in a way that is consistent both with evidence on balanced growth and evidence on the elasticity of substitution between capital and labor. The same would be true business-cycle analyses, which are also restricted to the Cobb-Douglas case when trying to understand the importance of investmentspecific technical change (e.g., Greenwood et al., 2000; Fisher, 2006; DiCecio, 2009). Development accounting could also benefit from models that allow for separate contributions from labor- and capital-augmenting technical change (Hsieh and Klenow, 2010; Schoellman, 2011). Work by Caselli and Feyrer (2007) also demonstrates the importance of including natural resources in development accounting exercises.

Separate from the accounting literate, models of economic growth with capital-augmenting technical change would be useful in the study of automation, artificial intelligence, and laborsaving technical change, topics that have recently received a great deal of attention in the growth literature (e.g., Benzell et al., 2015; Hémous and Olsen, 2016; Brynjolfsson and McAfee, 2012, 2014). An outstanding question in this literature is how to best model the impact of technology on labor market outcomes (Acemoglu and Autor, 2011; Acemoglu and Restrepo, 2016, 2018). Models with only one type of technology in the long run - e.g., those constrained the Uzawa (1961) theorem are naturally of limited use when trying to determine when technological change is worker-friendly and when it is not. In standard neoclassical production functions, capital- and labor-augmenting technical change have different impacts on the labor share of income. With low elasticities of substitution, positive shocks to labor- and capital-augmenting technologies also have opposite effects on the levels of wages and rental rates (Acemoglu, 2010; Acemoglu and Restrepo, 2018). Thus, including both capital- and labor-augmenting technical greatly increases the usefulness of these models in studying impacts of new technologies. ${ }^{53}$

\section{Conclusion}

The Uzawa (1961) steady state theorem has long posed significant problems for neoclassical models of balanced growth (Jones and Scrimgeour, 2008). As conventionally understood, the theorem

\footnotetext{
${ }^{52}$ See, He et al. (2008) and Maliar and Maliar (2011) for discussions of how to make these models consistent with balanced growth.

${ }^{53} \mathrm{On}$ a related note, much of the literature on automation has argued that factor-augmenting technologies are an ineffective way of modelling labor-saving technical change. Instead, such papers argue for using a task-based model along the lines of Zeira (1998) (e.g., Acemoglu and Autor, 2011; Acemoglu and Restrepo, 2016, 2018). However, as discussed in Acemoglu and Restrepo (2016), task-based growth models are still subject to the Uzawa theorem, as long as they can be represented by an aggregate production function. Indeed, Propositions 1 and 3 imply that these models have Uzawa and Factor-Augmenting representations on the BGP. To the best of our knowledge, no task-based model of endogenous growth has capital-augmenting technical change on the BGP.
} 
states that all technological change must be labor-augmenting on a balanced growth path, unless the production function is Cobb-Douglas. This constraint makes it impossible for the neoclassical model to be consistent with data indicating that (i) the two-factor aggregate production function is not Cobb-Douglas and (ii) the relative price of capital goods has been declining. This is a significant limitation. Substitution between capital and labor and technological change are the key forces that generate balanced growth in the standard model (see, e.g., Solow, 1956, 1994).

To provide insight into this apparent contradiction, we prove a multi-factor version of the Uzawa (1961) steady state theorem. The extended version of the theorem says that, on a balanced growth path, production can be represented by a neoclassical production function without capital-augmenting technical. As in the standard Uzawa theorem, this does not imply that the true production function does not have capital-augmenting technical change. More precisely, the theorem does not rule out the existence of multiple different representations of aggregate production along the balanced growth path. Thus, we also examine the circumstances under which there is a representation of the aggregate production function with capital-augmenting technical change. We prove that, with more than two factors of production, neoclassical production functions can accommodate capital-augmenting technical change, as long as there is a single factor than has a unit-elastic elasticity of substitution with reproducible capital.

This suggests a natural way to resolve the contradiction affecting more standard models: add more factors of production. In reality, there are many factors of production besides labor and reproducible capital. If any of these has a Cobb-Douglas relationship with capital, then multifactor neoclassical models can be consistent with the data. We also build a three-factor endogenous growth model and show that is converges to a balanced growth path with positive rates of capitalaugmenting technical change.

We strongly suggest that future empirical work investigates whether there is a factor of production that is Cobb-Douglas with reproducible capital. If such a factor can be identified, then tractable aggregate models can be made more consistent with data and will be better able to study a wide range of economic phenomena. 


\section{References}

Acemoglu, D. (1998). Why do new technologies complement skills? directed technical change and wage inequality. Quarterly Journal of Economics, pages 1055-1089.

Acemoglu, D. (2002). Directed technical change. Review of Economic Studies, 69(4):781-809.

Acemoglu, D. (2003). Labor-and capital-augmenting technical change. Journal of the European Economic Association, 1(1):1-37.

Acemoglu, D. (2008). Introduction to modern economic growth. Princeton University Press.

Acemoglu, D. (2010). When does labor scarcity encourage innovation? Journal of Political Economy, 118(6):1037-1078.

Acemoglu, D., Aghion, P., Bursztyn, L., and Hemous, D. (2012). The environment and directed technical change. American Economic Review, 102(1):131-66.

Acemoglu, D. and Autor, D. (2011). Skills, tasks and technologies: Implications for employment and earnings. Handbook of Labor Economics, 4:1043-1171.

Acemoglu, D. and Restrepo, P. (2016). The race between machine and man: Implications of technology for growth, factor shares and employment. NBER Working Paper.

Acemoglu, D. and Restrepo, P. (2018). Artificial intelligence, automation and work. NBER Working Paper.

Alvarez-Cuadrado, F., Van Long, N., and Poschke, M. (2018). Capital-labor substitution, structural change and the labor income share. Journal of Economic Dynamics and Control, 87:206-231.

Antras, P. et al. (2004). Is the us aggregate production function cobb-douglas? new estimates of the elasticity of substitution. Contributions to Macroeconomics, 4(1):1-34.

Ashraf, Q. and Galor, O. (2011). Dynamics and stagnation in the malthusian epoch. American Economic Review, 101(5):2003-41.

Barrage, L. (2019). Optimal dynamic carbon taxes in a climate-economy model with distortionary fiscal policy. Review of Economic Studies.

Barro, R. and Sala-i Martin, X. (2004). Economic Growth. MIT Press, 2 edition.

Benzell, S. G., Kotlikoff, L. J., LaGarda, G., and Sachs, J. D. (2015). Robots are us: Some economics of human replacement. Technical report, National Bureau of Economic Research. 
Bloom, N., Jones, C. I., Van Reenen, J., and Webb, M. (2017). Are ideas getting harder to find? NBER Working Paper.

Brynjolfsson, E. and McAfee, A. (2012). Race against the machine: How the digital revolution is accelerating innovation, driving productivity, and irreversibly transforming employment and the economy. Brynjolfsson and McAfee.

Brynjolfsson, E. and McAfee, A. (2014). The second machine age: Work, progress, and prosperity in a time of brilliant technologies. WW Norton \& Company.

Caselli, F. and Feyrer, J. (2007). The marginal product of capital. The quarterly journal of economics, 122(2):535-568.

Chirinko, R. S. (2008). $\sigma$ : The long and short of it. Journal of Macroeconomics, 30(2):671-686.

Chirinko, R. S., Fazzari, S. M., and Meyer, A. P. (2011). A new approach to estimating production function parameters: the elusive capital-labor substitution elasticity. Journal of Business $\mathcal{E}$ Economic Statistics, 29(4):587-594.

Cummins, J. G. and Violante, G. L. (2002). Investment-specific technical change in the united states (1947-2000): Measurement and macroeconomic consequences. Review of Economic dynamics, $5(2): 243-284$.

DiCecio, R. (2009). Sticky wages and sectoral labor comovement. Journal of Economic Dynamics and Control, 33(3):538-553.

Fisher, J. D. (2006). The dynamic effects of neutral and investment-specific technology shocks. Journal of political Economy, 114(3):413-451.

Galor, O. (2011). Unified growth theory. Princeton University Press.

Galor, O. and Weil, D. N. (2000). Population, technology, and growth: From malthusian stagnation to the demographic transition and beyond. American economic review, 90(4):806-828.

Golosov, M., Hassler, J., Krusell, P., and Tsyvinski, A. (2014). Optimal taxes on fossil fuel in general equilibrium. Econometrica, 82(1):41-88.

Greenwood, J., Hercowitz, Z., and Krusell, P. (1997). Long-run implications of investment-specific technological change. American Economic Review, pages 342-362.

Greenwood, J., Hercowitz, Z., and Krusell, P. (2000). The role of investment-specific technological change in the business cycle. European Economic Review, 44(1):91-115. 
Grossman, G. M., Helpman, E., Oberfield, E., and Sampson, T. (2017). Balanced growth despite uzawa. American Economic Review.

Growiec, J. (2007). Beyond the linearity critique: the knife-edge assumption of steady-state growth. Economic Theory, 31(3):489-499.

Hansen, G. D. and Prescott, E. C. (2002). Malthus to solow. American economic review, 92(4):12051217.

Hassler, J., Krusell, P., and Olovsson, C. (2016). Directed technical change as a response to natural-resource scarcity. Working Paper.

He, H., Liu, Z., et al. (2008). Investment-specific technological change, skill accumulation, and wage inequality. Review of Economic Dynamics, 11(2):314-334.

Heal, G. (1976). The relationship between price and extraction cost for a resource with a backstop technology. The Bell Journal of Economics, pages 371-378.

Hémous, D. and Olsen, M. (2016). The rise of the machines: Automation, horizontal innovation and income inequality. Working Paper.

Herrendorf, B., Herrington, C., and Valentinyi, A. (2015). Sectoral technology and structural transformation. American Economic Journal: Macroeconomics, 7(4):104-33.

Horii, R. and Iwaisako, T. (2007). Economic growth with imperfect protection of intellectual property rights. Journal of Economics, 90(1):45-85.

Hotelling, H. (1931). The economics of exhaustible resources. Journal of Political Economy, pages $137-175$.

Hsieh, C.-T. and Klenow, P. J. (2010). Development accounting. American Economic Journal: Macroeconomics, 2(1):207-23.

Irmen, A. (2016). A generalized steady-state growth theorem. Macroeconomic Dynamics, pages $1-26$.

Irmen, A. (2017). Capital-and labor-saving technical change in an aging economy. International Economic Review, 58(1):261-285.

Irmen, A. and Tabaković, A. (2017). Endogenous capital-and labor-augmenting technical change in the neoclassical growth model. Journal of Economic Theory, 170:346-384.

Jones, C. I. (2002). Sources of us economic growth in a world of ideas. American Economic Review, 92(1):220-239. 
Jones, C. I. (2005). The shape of production functions and the direction of technical change. Quarterly Journal of Economics, 120(2):517-549.

Jones, C. I. (2016). The facts of economic growth. Handbook of Macroeconomics, 2:3-69.

Jones, C. I. and Romer, P. M. (2010). The new kaldor facts: ideas, institutions, population, and human capital. American Economic Journal: Macroeconomics, 2(1):224-245.

Jones, C. I. and Scrimgeour, D. (2008). A new proof of uzawa's steady-state growth theorem. Review of Economics and Statistics, 90(1):180-182.

Kaldor, N. (1961). Capital accumulation and economic growth. In The theory of capital, pages 177-222. Springer.

Karabarbounis, L. and Neiman, B. (2014). The global decline of the labor share. The Quarterly Journal of Economics, 129(1):61-103.

Klump, R., McAdam, P., and Willman, A. (2007). Factor substitution and factor-augmenting technical progress in the united states: a normalized supply-side system approach. The Review of Economics and Statistics, 89(1):183-192.

Krusell, P. (1998). Investment-specific $\mathrm{r}$ and $\mathrm{d}$ and the decline in the relative price of capital. Journal of Economic Growth, 3(2):131-141.

Krusell, P., Ohanian, L. E., Ríos-Rull, J.-V., and Violante, G. L. (2000). Capital-skill complementarity and inequality: A macroeconomic analysis. Econometrica, 68(5):1029-1053.

Leon-Ledesma, M. A. and Satchi, M. (2016). Appropriate technology and balanced growth. The Review of Economic Studies.

Li, D., Huang, J., et al. (2016). A variant of uzawassteady-state theorem in a malthusian model. Economics Bulletin, 36(3):1371-1375.

Maliar, L. and Maliar, S. (2011). Capital-skill complementarity and balanced growth. Economica, 78(310):240-259.

Mas-Colell, A., Whinston, M. D., Green, J. R., et al. (1995). Microeconomic theory, volume 1. Oxford university press New York.

Oberfield, E. and Raval, D. (2014). Micro data and macro technology. Technical report, National Bureau of Economic Research.

Papell, D. H. and Prodan, R. (2014). Long run time series tests of constant steady-state growth. Economic Modelling, 42:464-474. 
Piketty, T. (2014). Capital in the 21st Century. Harvard University Press Cambridge, MA.

Schlicht, E. (2006). A variant of uzawa's theorem. Economics Bulletin, 5(6):1-5.

Schoellman, T. (2011). Education quality and development accounting. The Review of Economic Studies, 79(1):388-417.

Smulders, S. and De Nooij, M. (2003). The impact of energy conservation on technology and economic growth. Resource and Energy Economics, 25(1):59-79.

Solow, R. M. (1956). A contribution to the theory of economic growth. The quarterly journal of economics, 70(1):65-94.

Solow, R. M. (1994). Perspectives on growth theory. Journal of Economic Perspectives, 8(1):45-54.

Swan, T. W. (1956). Economic growth and capital accumulation. Economic record, 32(2):334-361.

Uzawa, H. (1961). Neutral inventions and the stability of growth equilibrium. Review of Economic Studies, 28(2):117-124.

Valentinyi, A. and Herrendorf, B. (2008). Measuring factor income shares at the sectoral level. Review of Economic Dynamics, 11(4):820-835.

Zeira, J. (1998). Workers, machines, and economic growth. The Quarterly Journal of Economics, 113(4):1091-1117. 


\section{A Appendix}

\section{A.1 Data}

\section{A.1.1 Figure 1}

Real GDP. U.S. Bureau of Economic Analysis, Real Gross Domestic Product [GDPCA], retrieved from FRED, Federal Reserve Bank of St. Louis; https://fred.stlouisfed.org/series/GDPCA, February 15, 2019. Shown in Panel (a).

Investment. U.S. Bureau of Economic Analysis, Real Gross Private Domestic Investment [GPDIC1], retrieved from FRED, Federal Reserve Bank of St. Louis; https://fred.stlouisfed.org/series/GPDIC1, February 15, 2019. Shown in Panel (a).

Consumption. U.S. Bureau of Economic Analysis, Real Personal Consumption Expenditures [PCECC96], retrieved from FRED, Federal Reserve Bank of St. Louis; https://fred.stlouisfed.org/series/PCECC96 February 15, 2019. Shown in Panel (a).

Nominal Capital Stock. U.S. Bureau of Economic Analysis, Current-Cost Net Stock of Fixed Assets, Fixed Asset Table 1.1. Not shown in Table.

GDP Deflator. Bureau of Economic Analysis, Implicit Price Deflator for Gross Domestic Product, NIPA Table 1.1.9. Not shown in Table.

Real Capital Stock $(K)$. Nominal Capital Stock/GDP Deflator. Shown in Panel (a).

Investment Deflator. Bureau of Economic Analysis, Implicit Price Deflator for Gross Private Domestic Investment, NIPA Table 1.1.9. Not shown in Table.

Non-Residential Investment Deflator. Bureau of Economic Analysis, Implicit Price Deflator for Fixed, Non-Residental Investment, NIPA Table 1.1.9. Not shown in Table.

Equipment Investment Deflator. Bureau of Economic Analysis, Implicit Price Deflator for Equipment, NIPA Table 1.1.9. Not shown in Table.

Personal Consumption Expenditures Deflator. Bureau of Economic Analysis, Implicit Price Deflator for Personal Consumption Expenditures, NIPA Table 1.1.9. Not shown in Table. 
Relative Price of Investment (various). Investment Deflator/Consumption Deflator. Applied to all investment, non-residential, and equipment deflators. Shown in panel (b).

\section{A.1.2 Table 1}

Energy Expenditure Share. Energy expenditure as a share of GDP (\%). Data available from 1970-2014. Source: 'Table 1.5: Energy consumption, expenditures, and emissions indicators estimates, 1949-2011' at https://www.eia.gov/totalenergy/data/annual/.

\section{A.1.3 Calibration}

See information for Relative Price of Investment Goods, Nominal Capital Stock, and Real GDP from above.

Nominal GDP. U.S. Bureau of Economic Analysis, Gross Domestic Product [GDP], retrieved from FRED, Federal Reserve Bank of St. Louis; https://fred.stlouisfed.org/series/GDP, February $15,2019$.

R\&D Expenditure. U.S. Bureau of Economic Analysis, Gross Domestic Product: Research and Development [Y694RC1Q027SBEA], retrieved from FRED, Federal Reserve Bank of St. Louis; https://fred.stlouisfed.org/series/Y694RC1Q027SBEA, February 15, 2019.

Capital-Output Ratio. Nominal Capital Stock/Nominal GDP.

Labor Compensation. Bureau of Economic Analysis, (Compensation of Employees) + ( Proprietors' income with inventory valuation and capital consumption adjustments), NIPA Table 2.1.

Labor Share of Income. Labor Compensation / Nominal GDP.

R\&D Share of GDP. R\&D Expenditure / Nominal GDP.

Savings Rate. (Investment Share of GDP) - (R\&D Share of GDP). 


\section{A.2 Proofs}

\section{A.2.1 Proposition 2}

Proof. Note that the share of factor $Z_{t} \in\left\{K_{t}, X_{1, t}, \ldots, X_{J, t}\right\}$ is the same in period $t$ and 0 . Because the production function in period 0 is $F(\cdot ; 0) \equiv \widetilde{F}(\cdot)$,

$$
s_{Z}=F_{Z}\left(K_{t}, X_{1, t}, \ldots, X_{J, t} ; t\right) \frac{Z_{t}}{Y_{t}}=\widetilde{F}_{Z}\left(K_{0}, X_{1,0}, \ldots, X_{J, 0}\right) \frac{Z_{0}}{Y_{0}},
$$

where $\widetilde{F}_{Z}(\cdot)$ represents the derivative of function $\widetilde{F}(\cdot)$ with respect to its argument (see footnote 16). Note that, since function $\widetilde{F}(\cdot)$ has constant returns to scale, its partial derivative function $\widetilde{F}_{Z}(\cdot)$ must be homogeneous of degree 0 (See Theorem M.B.1 in Mas-Colell et al., 1995). Therefore, the value of $\widetilde{F}_{Z}(\cdot)$ will be unchanged when all of its arguments are multiplied to the same proportion $g^{t}=Y_{t} / Y_{0}=K_{t} / K_{0}=\widetilde{A}_{X j, t} X_{j, t} / X_{j, 0}$ (Here we used the stationarity of $K_{t} / Y_{t}$ from Lemma 1 ). Also, because the effective amount of production factors and the output grow at the same speed, $Z_{0} / Y_{0}=\widetilde{A}_{Z, t} Z_{t} / Y_{t}$ holds on the BGP (In the case of $Z_{t}=K_{t}$, we define $\widetilde{A}_{K, t} \equiv 1$ ). Therefore,

$$
s_{Z}=\widetilde{F}_{Z}\left(K_{t}, \widetilde{A}_{X 1, t} X_{1, t}, \ldots, \widetilde{A}_{X J, t} X_{J, t}\right) \widetilde{A}_{Z, t} \frac{Z_{t}}{Y_{t}}=\frac{\partial \widetilde{F}\left(K_{t}, \widetilde{A}_{X 1, t} X_{1, t}, \ldots, \widetilde{A}_{X J, t} X_{J, t}\right)}{\partial Z_{t}} \frac{Z_{t}}{Y_{t}}
$$

where the validity of the second equality is guaranteed by the chain rule (See footnote 17). By comparing the latter with (A.1), we obtain (6).

\section{A.2.2 Lemma 2}

Proof. If $j^{*}=0, \alpha=0$ follows. Then, the lemma becomes trivial, because the RHS of (10) is simply the original $F(\cdot ; 0)$ function. Therefore, we focus on the case of $j^{*} \geq 1$.

Let us consider a $K-X_{1}$ space keeping other inputs $\mathbf{X}_{-1} \equiv\left\{X_{2}, \ldots, X_{J}\right\}$ constant. Note that, since $\sigma_{K X 1,0}=1$, Definition 5 means that $d \ln \left(F_{K} / F_{X_{1}}\right) / d \ln \left(K / X_{1}\right)=-1$ holds on this $K-X_{1}$ space. Integrating this differential equation gives $\ln \left(F_{K} / F_{X_{1}}\right)=-\ln \left(K / X_{1}\right)+\widetilde{\xi}_{1}$, where $\widetilde{\xi}_{1}$ is a constant of integration. Taking the exponential of the both sides gives $F_{K} / F_{X_{1}}=\left(X_{1} / K\right) \widehat{\xi}_{1}$, where $\widehat{\xi}_{1} \equiv \exp \widetilde{\xi}_{1}>0$ is a positive constant.

Now, consider the isoquant curve of production function $F_{K}\left(K, X_{1}, \ldots, X_{J} ; 0\right)$ on this $K-X_{1}$ space. The slope of the isoquant curve is $d X_{1} / d K=-F_{K} / F_{X_{1}}=\left(X_{1} / K\right) \widehat{\xi}_{1}$. Integrating this differential equation gives $\ln K=-\widehat{\xi}_{1} \ln X_{1}+\widehat{\kappa}_{1}$, where $\widehat{\kappa}_{1}$ is another constant of integration. By taking exponential, the isoquant curve is written as $K=\kappa_{1} X_{1}^{-\widehat{\xi}_{1}}$, where $\kappa_{1} \equiv \exp \widehat{\kappa}_{1}>0$ is a positive constant.

Let us focus on the isoquant curve that runs through the allocation at time $t=0$; i.e., $\left\{K_{0}, \mathbf{X}_{0}\right\}$. The constant $\kappa_{1}$ representing this curve is obtained by substituting $K=K_{0}$ and $X_{1}=X_{1,0}$ into above expression, yielding $K=K_{0}\left(X_{1,0} / X_{1}\right)^{\widehat{\xi}_{1}}$. By substituting $X_{1}=1$ into 
this curve, we know that the allocation with $K=K_{0} X_{0}^{\xi_{1,0}}$ and $X_{1}=1$ gives the same amount of output with that with $K=K_{0}$ and $X_{1}=X_{1,0}$, keeping other inputs constant. This means $Y_{0}=F\left(K_{0} X_{0}^{\widehat{\xi}_{1}}, 1, X_{2,0}, \ldots, X_{J, 0} ; 0\right)$.

Repeating this procedure for $j=1, \ldots, j^{*}$, we obtain

$$
Y_{0}=F(K_{0} \prod_{j=1}^{j^{*}}\left(X_{j, 0}\right)^{\widehat{\xi}_{j}}, \underbrace{1, \ldots, 1}_{j^{*}}, X_{j^{*}+1,0}, \ldots, X_{J, 0} ; 0) .
$$

Define $\alpha \equiv\left(1+\sum_{j=1}^{j^{*}} \widehat{\xi}_{j}\right)^{-1}$ and $\xi_{j}=\alpha \widehat{\xi}_{j}$ for $j=\left\{1, \ldots, j^{*}\right\}$. Then, using the definition of $\widehat{F}(\cdot)$ in (11), it can be confirmed that (A.2) is the same as (10).

Lastly, we confirm that $\widehat{F}(\cdot)$ has constant returns to scale. Multiplying all the arguments in $\widehat{F}\left(z_{0}, z_{j^{*}+1}, \ldots, z_{J}\right)$ by $\lambda>0$ gives $\widehat{F}\left(\lambda z_{0}, \lambda z_{j^{*}+1}, \ldots, \lambda z_{J}\right)=F\left(\left(\lambda z_{0}\right)^{1 / \alpha}, 1, \ldots, 1, \lambda z_{j^{*}+1}, \ldots, \lambda z_{J} ; 0\right)$. Note that, from the definition of $\alpha,\left(\lambda z_{0}\right)^{1 / \alpha}=\lambda^{1+\sum_{j=1}^{j^{*}} \widehat{\xi}_{j}} z_{0}^{1 / \alpha}=\lambda z_{0}^{1 / \alpha} \prod_{j=1}^{j^{*}} \lambda^{\widehat{\xi}_{j}}$. Also, recall that the isoquant curve of $F(\cdot ; 0)$ on a $K-X_{j}$ space takes the form of $K=\kappa_{j} X_{j}^{-\widehat{\xi}_{j}}$ for $j=$ $1, \ldots, j^{*}$, which means that if we multiply $X_{j}$ by $\lambda$ and $K$ by $\lambda^{-\widehat{\xi}_{j}}$ then the output will be unchanged. Using these, $F\left(\left(\lambda z_{0}\right)^{1 / \alpha}, 1, \ldots, 1, \lambda z_{j^{*}+1}, \ldots, \lambda z_{J} ; 0\right)=F(\lambda z_{0}^{1 / \alpha}, \underbrace{\lambda, \ldots, \lambda}_{j^{*}}, \lambda z_{j^{*}+1}, \ldots, \lambda z_{J} ; 0)=$ $\lambda F(z_{0}^{1 / \alpha}, \underbrace{1, \ldots, 1}_{j^{*}}, z_{j^{*}+1}, \ldots, z_{J} ; 0)$, where the last equality follows because $F(\cdot ; 0)$ has constant returns to scale. The last expression coincides with $\lambda \widehat{F}\left(z_{0}, z_{j^{*}+1}, \ldots, z_{J}\right)$. This confirms the constant-returnsto-scale property of $\widehat{F}(\cdot)$.

\section{A.2.3 Proposition 3}

Proof. From Lemma 1 and condition (12), it can be confirmed that the first argument in function $\widehat{F}(\cdot)$ in (13) is multiplied by $g$ each period. The same holds for the other arguments: $A_{X j, t} X_{j, t}$ for $j=j^{*}+1, \ldots, J$. Since $\widehat{F}(\cdot)$ has constant returns to scale, the RHS of (13) is multiplied by $g$ each period. In period $0,(7)$ holds because it is identical with (10). Since $Y_{t}$ is multiplied by $g$ every period by the definition of the BGP, (13) holds for all $t \geq 0$.

\section{A.2.4 Proposition 4}

Proof. Let us first consider the case of $Z_{t}=K_{t}$. Note that the share of capital is the same in period $t$ and 0 . Because the production function in period 0 can be represented in the form of (10),

$$
s_{K}=F_{K}\left(K_{t}, X_{1, t}, \ldots, X_{J, t} ; t\right) \frac{K_{t}}{Y_{t}}=\frac{\partial}{\partial K_{0}} \widehat{F}\left(K_{0}^{\alpha} \prod_{j=1}^{j^{*}}\left(X_{j, 0}\right)^{\xi_{j}}, X_{j^{*}+1,0}, \ldots, X_{J, 0}\right) \frac{K_{0}}{Y_{0}}
$$


Define $\widehat{F}_{M}(\cdot)$ as the derivative of function $\widehat{F}(\cdot)$ with respect to its first argument. In (A.3), the first argument is the capital composite, $M_{0}=K_{0}^{\alpha} \prod_{j=1}^{j^{*}}\left(X_{j, 0}\right)^{\xi_{j}}$. Then, using the chain rule, the RHS of (A.3) becomes

$$
s_{K}=\widehat{F}_{M}\left(M_{0}, X_{j^{*}+1,0}, \ldots, X_{J, 0}\right) \frac{d M_{0}}{d K_{0}} \frac{K_{0}}{Y_{0}} .=\widehat{F}_{M}\left(M_{0}, X_{j^{*}+1,0}, \ldots, X_{J, 0}\right) \frac{\alpha M_{0}}{Y_{0}}
$$

Since $\widehat{F}(\cdot)$ has constant returns to scale from Lemma 2, its derivative $\widehat{F}_{M}(\cdot)$ is a homogeneous function of degree 0 . Recall that, from Lemma 1 and condition (12), $M_{t}$ is multiplied by $g$ every period. The same applies to $A_{X j, t} X_{j, t}$ for $j=j^{*}+1, \ldots, J$. Therefore, every argument in $\widehat{F}_{M}\left(M_{t}, A_{X j^{*}+1, t} X_{j^{*}+1, t}, \ldots, A_{X J, t} X_{J, t}\right)$ is multiplied by $g$ every period, which does not change the value of $F_{M}(\cdot)$ over time. Also $\alpha M_{0} / Y_{0}=\alpha M_{t} / Y_{t}=\left(\alpha M_{t} / K_{t}\right)\left(K_{t} / Y_{t}\right)$ holds because $M_{t}$ and $Y_{t}$ grow at the same speed. From these and the chain rule,

$$
\begin{aligned}
s_{K} & =\widehat{F}_{M}\left(M_{t}, A_{X j^{*}+1, t} X_{j^{*}+1, t}, \ldots, A_{X J, t} X_{J, t}\right) \frac{\alpha M_{t}}{k_{t}} \frac{\alpha K_{t}}{Y_{t}} \\
& =\frac{\partial \widehat{F}\left(M_{t}, A_{X j^{*}+1, t} X_{j^{*}+1, t}, \ldots, A_{X J, t} X_{J, t}\right)}{\partial Z_{t}} \frac{\alpha K_{t}}{Y_{t}},
\end{aligned}
$$

where $M_{t}=\left(A_{K, t} K_{t}\right)^{\alpha} \prod_{j=1}^{j^{*}}\left(A_{X j, t} X_{j, t}\right)$. By comparing (A.3) with (A.5), we obtain (14) for the case of $Z_{t}=K_{t}$.

The proof of the proposition for the case of $Z_{t}=X_{j, t}, j \in\left\{1, \ldots, j^{*}\right\}$, follows the same pattern as in (A.3)-(A.5), with only the modification that $K_{t}$ is replaced by $X_{j, t}$ and $\alpha$ by $\xi_{j}$.

Finally, the the case of $Z_{t}=X_{j, t}, j \in\left\{j^{*}, \ldots, J\right\}$, can be confirmed in the same way as in Proposition 2 , by swapping $\widetilde{F}(\cdot)$ with $\widehat{F}(\cdot)$. In this case, we define $\widehat{F}_{X j}(\cdot), j \in\left\{j^{*}, \ldots, J\right\}$, as the derivative of function $\widehat{F}(\cdot)$ with respect to its $\left(j-j^{*}+1\right)$ th argument. ${ }^{54}$

\section{A.2.5 Lemma 3}

Proof. Consider a BGP. We will show that $\mu_{t}, k_{t}$ and $c_{t}$ must be constant in turn. First, from the definition of a BGP, $N_{t+1} / N_{t}=\left(A_{L, t+1} L_{t+1}\right) /\left(A_{L, t} L_{t}\right)=\widehat{\gamma}_{L}\left(\mu_{t+1}\right) g_{L}$ is constant. To keep the RHS of the latter equation constant, $\mu_{t}$ must also be constant, since $\widehat{\gamma}_{L}(\cdot)$ is a strictly increasing function from Proposition 5. Second, since growth factors of $C_{t}$ and $N_{t}$ are constant, the growth factor of $c_{t}=C_{t} / A_{t} L_{t}=C_{t} / N_{t}$ is also constant. This, in turn, means that the LHS of the Euler equation (48) is constant. Then, for the RHS of (48) to be constant, $k_{t}$ must be constant, since we already know that $\mu_{t}$ is constant as shown above. Similarly, the growth factor of $k_{t}=K_{t} / A_{t} L_{t}=K_{t} / N_{t}$ is constant on the BGP, which means the LHS of (46) is constant. For its RHS to be constant, given that $\mu_{t}$ and $k_{t}$ are already shown to be constant, $c_{t}$ also needs to be constant.

\footnotetext{
${ }^{54}$ Recall that $j^{*}$ arguments are eliminated from function $\widehat{F}(\cdot)$ in definition (11).
} 